\title{
Breast milk alkylglycerols sustain beige adipocytes through adipose tissue macrophages
}

\author{
Haidong Yu, ${ }^{1}$ Sedat Dilbaz, ${ }^{1,2}$ Jonas Coßmann, ${ }^{1}$ Anh Cuong Hoang, ${ }^{1}$ Victoria Diedrich, ${ }^{1}$ Annika Herwig, ${ }^{1}$ Akiko Harauma, ${ }^{3}$ \\ Yukino Hoshi, ${ }^{3}$ Toru Moriguchi, ${ }^{3}$ Kathrin Landgraf, ${ }^{4}$ Antje Körner, ${ }^{4}$ Christina Lucas, ${ }^{5}$ Susanne Brodesser, ${ }^{5}$ Lajos Balogh, ${ }^{6}$ \\ Julianna Thuróczy, ${ }^{6}$ Gopal Karemore, ${ }^{7}$ Michael Scott Kuefner, ${ }^{8}$ Edwards A. Park, ${ }^{8}$ Christine Rapp, ${ }^{9}$ \\ Jeffrey Bryant Travers, ${ }^{9}$ and Tamás Röszer ${ }^{1}$ \\ IInstitute of Neurobiology, and Institute of Comparative Molecular Endocrinology, University of Ulm, Ulm, Germany. ${ }^{3}$ Department of Food and Life Science, Azabu University, Sagamihara, Kanagawa, Japan. \\ ${ }^{4}$ Center for Pediatric Research, University Hospital for Children and Adolescents, University of Leipzig, Leipzig, Germany. ${ }^{5}$ Lipidomics Facility, CECAD Research Center, University of Cologne, Cologne, Germany. \\ ${ }^{6}$ Department of Nuclear Medicine, National Public Health Center (NPHC), Budapest, Hungary. ${ }^{7}$ Novo Nordisk Foundation Center for Protein Research, University of Copenhagen, Copenhagen, Denmark. \\ ${ }^{8}$ Veterans Affairs Medical Center and the Department of Pharmacology, University of Tennessee Health Science Center, Memphis, Tennessee, USA. ${ }^{9}$ Department of Pharmacology and Toxicology, \\ Wright State University, Dayton, Ohio, USA.
}

Prevalence of obesity among infants and children below 5 years of age is rising dramatically, and early childhood obesity is a forerunner of obesity and obesity-associated diseases in adulthood. Childhood obesity is hence one of the most serious public health challenges today. Here, we have identified a mother-to-child lipid signaling that protects from obesity. We have found that breast milk-specific lipid species, so-called alkylglycerol-type (AKG-type) ether lipids, which are absent from infant formula and adult-type diets, maintain beige adipose tissue (BeAT) in the infant and impede the transformation of BeAT into lipid-storing white adipose tissue (WAT). Breast milk AKGs are metabolized by adipose tissue macrophages (ATMs) to platelet-activating factor (PAF), which ultimately activates IL-6/STAT3 signaling in adipocytes and triggers BeAT development in the infant. Accordingly, lack of AKC intake in infancy leads to a premature loss of BeAT and increases fat accumulation. AKC signaling is specific for infants and is inactivated in adulthood. However, in obese adipose tissue, ATMs regain their ability to metabolize AKGs, which reduces obesity. In summary, AKGs are specific lipid signals of breast milk that are essential for healthy adipose tissue development.

\section{Introduction}

Adipose tissue (AT) is a multifunctional organ that, beyond its traditional role as a lipid storage site, is pivotal in metabolic and endocrine health (1). Excess development of AT, however, leads to obesity and is a forerunner of several chronic diseases. It is estimated that $38 \%$ of the world's adult population will be overweight by 2030 and another $20 \%$ will be obese $(2,3)$. Obesity-related diseases are among the fastest growing noncommunicable diseases today. Worryingly, the incidence of obesity is increasing among infants and children below 5 years of age, showing a $30 \%$ increase globally in the last 2 decades and a 10-fold increase in the last 4 decades (2). This translates to 41 million obese infants and young children in 2016, and this is estimated to rise to 70 million in the next 6 years (2). Most recent studies show that obesity status in early childhood is a key determinant of obesity development in later life, and being obese as a child increases the risk of obesity-

\section{Related Commentary: p. 2198}

Authorship note: HY and SD contributed equally to this work. Conflict of interest: GK has an affiliation with Novo Nordisk A/S Copyright: () 2019, American Society for Clinical Investigation. Submitted: October 30, 2018; Accepted: March 12, 2019; Published: May 13, 2019. Reference information: / Clin Invest. 2019;129(6):2485-2499. https://doi.org/10.1172/JCl125646. related diseases in adulthood $(3,4)$. Accordingly, childhood obesity is one of the most serious public health challenges of our time (5).

Recent studies show that children who are breastfed have a lower probability of developing obesity (6-8), but how this occurs remains unknown (9). Breast milk is a rich source of lipid mediators (10), and lipid signaling is essential for AT physiology $(1,11)$. Indeed, breast milk lipid composition is mirrored by AT lipid composition (12) and AT is a major source of breast milk lipids (13). Functions of breast milk lipid mediators have been mostly studied in the context of neonate immunity and the inflammatory state (8, 14-17), and it is much less understood how breast milk-derived lipid signals control AT development.

Neonates are born with beige AT-dominated (BeAT-dominated) AT depots, which in part support the maintenance of the body's core temperature (18). BeAT shares ontogeny with white AT (WAT); however, it has some functional similarities to brown AT (BAT), such as increased mitochondrial content, multilocular storing of lipid droplets, and the ability to burn off lipids as heat due to an uncoupling mitochondrial terminal electron transport chain from ATP generation (18). BeAT is progressively replaced by WAT in late infancy and in adolescence $(18,19)$. This replacement of BeAT by WAT is accelerated in obese children (20), showing that premature loss of BeAT can be a cause of obesity. Along this line, increasing WAT/BeAT transformation in adulthood has been recently identified as a potential strategy for treating obesity (18, 
21). However, the signals that determine BeAT content and set the point of BeAT/WAT transformation in infancy are unclear.

We show here that a breast milk-specific lipid group, the so-called alkylglycerol (AKG, 1-O-alkyl-sn-glycerol) ether lipids, sustain infant BeAT. AT macrophages (ATMs) metabolize AKGs, and this activates adipocyte STAT3 signaling and triggers BeAT development. Lack of AKGs causes premature BeAT/WAT transformation. AKGs are hence powerful mediators of breast milk and they establish a mother-to-child lipid signaling bridge that is pivotal for healthy AT development.

\section{Results}

Breast milk is the only relevant nutritional source of AKGs. AKGs of human breast milk are chimyl alcohol (CA), batyl alcohol (BA), and selachyl alcohol (SA); these are present in micromolar concentrations (22-25) (Figure 1A and Supplemental Figure 1; supplemental material available online with this article; https://doi.org/10.1172/ JCI125646DS1). Breast milk is the sole relevant source of AKGs in human nutrition, and it is highly improbable that a human adult consumes the equivalent of the daily AKG intake of a breastfed infant (Supplemental Figure 2, A-C). To determine whether AKGs are secreted into mouse's milk, we established a nanoelectrospray ionization tandem mass spectrometry-based analytic technique (Supplemental Figure 3, A-D). Mouse milk contained AKGs in the micromolar range (Figure 1, B and C, and Supplemental Figure $3 \mathrm{E})$. In accordance with previous studies $(22,23)$, we also found that human milk was rich in AKGs and, similarly, canine and goat milk contained AKGs (Supplemental Figure 3F). In contrast, AKGs were negligible in cow milk, the major component of infant formula (Supplemental Figure 3F).

Using the same approach, we also questioned whether AKGs were present in the blood plasma of neonate mice, finding that plasma AKG levels mirrored those in breast milk (Figure $1 \mathrm{C}$ and Supplemental Table 1). In contrast, AKGs were absent (CA and SA) or negligible (BA) in the plasma of adult mice (Figure $1 \mathrm{C}$ ). This suggests that breast milk was the source of AKGs. Consistently, when mice were fed AKGs, the plasma AKG level was increased, proving the intestinal absorption of AKGs (Supplemental Figure 3G). AKGs are absorbed by the lymphatic vessels of the intestine and are distributed in the systemic circulation by chylomicrons and low-density lipoproteins (Supplemental Figure 4A). AKGs can hence reach AT directly, without passing through the portal vein (Supplemental Figure $4 \mathrm{~B}$ ). This and the lipophilic nature of AKGs make it plausible that AKGs are deposited in AT (Supplemental Figure 4C). Accordingly, we found AKG accumulation in mouse and human AT (Supplemental Figure 4, D and E). While little is known about the metabolism of AKGs, an AKG-catabolizing enzyme, a so-called AKG-monooxygenase (AGMO or TMEM195; EC 1.14.16.5), has been recently identified $(26,27)$. AGMO is a membrane-bound enzyme that cleaves AKGs into glycerol and fatty aldehyde (FA) (Supplemental Figure $4 \mathrm{~F})(26,28)$. Transcriptional analysis of Agmo showed that its expression in the AKG-accumulating AT was moderate. However, liver, which had negligible AKG content, expressed much higher Agmo than any other organ tested (Supplemental Figure 4, G-J).

Breast milk AKGs maintain beige adipocytes in infant AT. Our data show that AKG intake is confined to infancy and that AKGs are enriched in AT, suggesting that they may affect AT physiology in infants. To test this, we increased the AKG intake in C57BL/6 neonate mice by approximately $20 \%$ between P3 and P10 (Figure 1D) by oral administration, using AKG-free olive oil as a vehicle (Supplemental Figure 3F). Littermate controls were fed with vehicle. P3-P10 is an important period for determination of body adiposity, since BeAT starts to develop into lipid-storing WAT (Supplemental Figure 5A). The human equivalent of this period is late infancy, when intensive AT remodeling takes place (29), and AT mass of the young child determines obesity status in later life (3). BeAT content, along with Ucp1 transcription, declines further from P10 to adulthood in mouse (Supplemental Figure 5B).

We found that the AKG-treated group had reduced inguinal AT (iAT) weight (Figure 1E) and retained a BeAT-dominated iAT (Figure 1, F-H). Adipocyte size and triacylglycerol (TG) content of iAT were significantly lower in AKG-treated mice than in vehicle-treated counterparts, whereas mitochondrial content, BeAT area, and plasma glycerol levels were significantly higher (Figure 1I), hallmarking BeAT development (30). Lean weight was not affected by AKGs (Figure 1I). Consistently, AKG treatment increased iAT transcription of Ucp1, Ppargc1a, Cox7a1, Cidea, Dio2, and Tmem 26 (Figure 1J and Supplemental Figure 5B), which are all markers of BeAT $(21,31)$. In contrast, no changes were detected in the transcription of $A d r b 3$ and its downstream targets Lipe (Hsl) and Pnpla2 (Atgl), which are responsible for lipolysis (Figure 1J). Moreover, AKG-treated mice challenged with hypothermia maintained a higher core temperature than the vehicle-treated mice (Supplemental Figure 5C). AKGs specifically affected iAT, the main site of BeAT development, whereas BAT was only moderately affected by AKG treatment (Supplemental Figure 5, D-G). AKGs did not affect weight or impose changes on other organs (Supplemental Figure 5, $\mathrm{H}-\mathrm{M}$ ). After weaning, AKG-treated mice had a reduced iAT mass, without changes in lean body weight (Supplemental Figure $5 \mathrm{~N}$ ).

Overall, our findings suggest that AKGs, which are taken up from breast milk, increase BeAT content. This raises the question as to whether the lack of breastfeeding impairs BeAT development. To test this possibility, we analyzed samples of inguinal subcutaneous AT from 20 human infants aged 0.2-1.0 year for UCP1 expression and BeAT content (Figure 2, A-D, and Supplemental Table 2). We found that if breastfeeding was the dominant nutritional source (Supplemental Figure 6A), UCP1 had peak levels at ages 0.2-0.3 year, with moderate levels from 0.4 year onwards (Figure $2 \mathrm{~A}$ ). In contrast, UCP1 mRNA expression was undetectable in most infants who were never breastfed or received negligible breastfeeding (Figure 2B, $P$ $=0.0019$, Mann-Whitney $U$ test, Supplemental Table 2). Similarly, when we compared exclusively breastfed, partial breastfed, and never breastfed infants, we found compromised or undetectable UCP1 levels in infants who received breastfeeding for $30 \%$ or more of their actual age or were never breastfed (Supplemental Figure 6A). When age-matched infants were compared, breastfed infants had higher UCP1 mRNA levels than formula-fed infants (Figure 2C; $P=0.0122$, Mann-Whitney $U$ test). Consistently, BeAT content was higher (Figure 2D), and mitochondria-rich, multilocular adipocytes were abundant in the AT of breastfed infants (Supplemental Figure 6B).

Formula-fed infants received cow milk-based, hence AKGdeficient, formula, which prompted us to examine the effect of AKG-deficient postnatal nutrition on BeAT. For this analysis, we tested 3 groups of mice: one received regular breastfeeding from 
<smiles>CCOCC(O)CO</smiles>

CA<smiles>CC(F)(F)OCC(O)CO</smiles>

BA<smiles>CCCCCOCC(O)CO</smiles>

SA
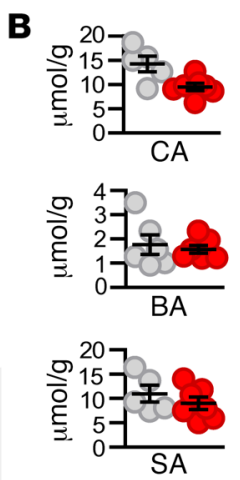

P3
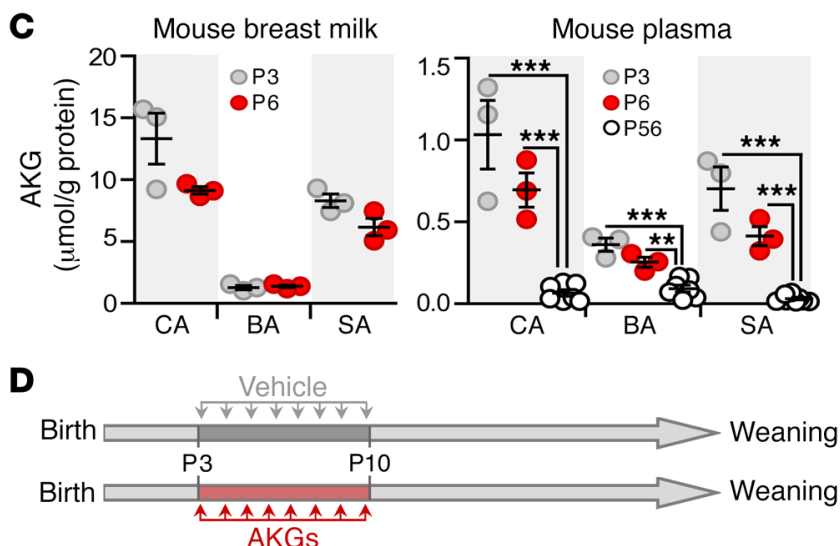

E

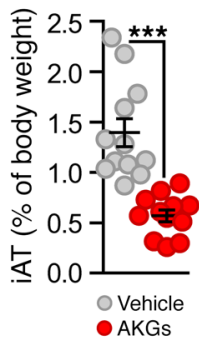

F

H\&E

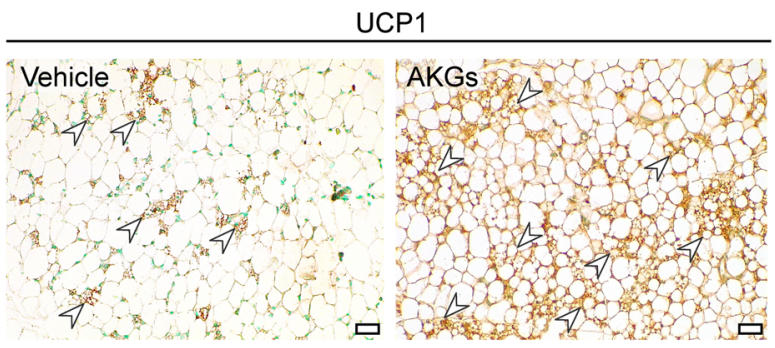

\section{G}

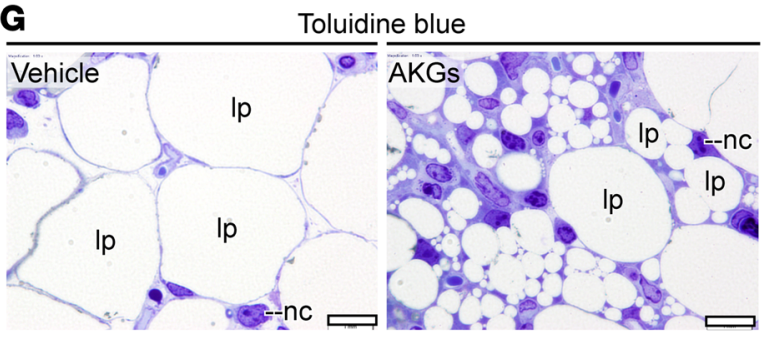

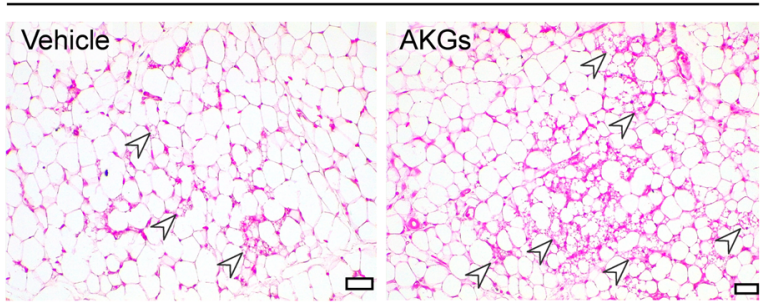
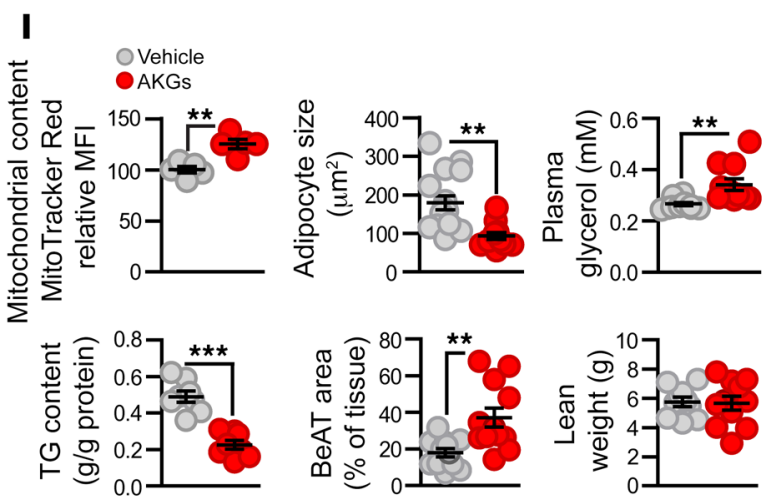

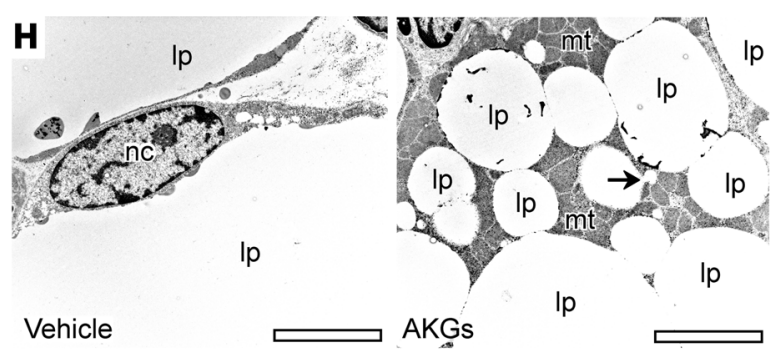

J

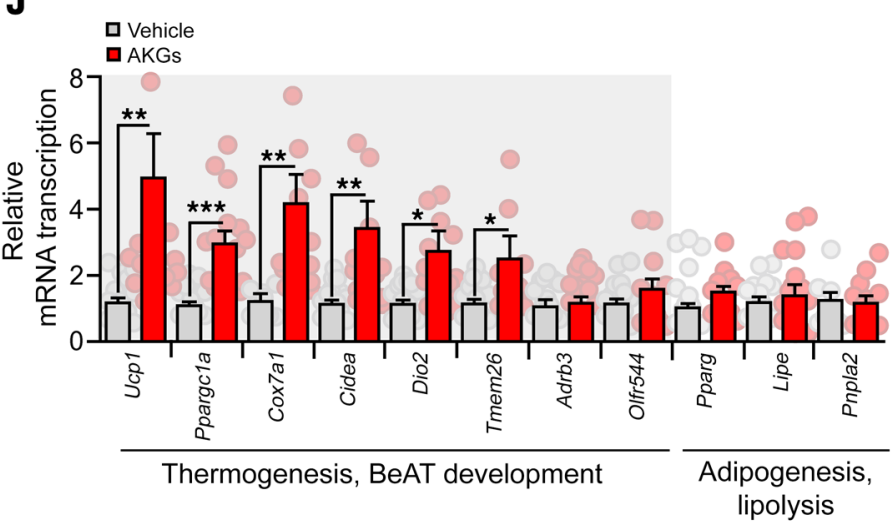

Figure 1. Breast milk AKGs increase beige adipocyte content in infant mice. (A) Structure of AKGs found in human breast milk. (B) Level of AKGs in mouse breast milk on P3 and P6 normalized to protein content. (C) Levels of AKGs in breast milk of 3 lactating mice and the plasma AKG levels of their respective litters on P3 and P6. One data point represents plasma pooled from 8 to 9 infant mice. Plasma AKC levels on P56. (D) Schematic of AKC treatment of infant mice. (E) iAT weight on P10 of vehicle- or AKG-treated mice. (F-H) Histology of iAT. H\&E staining and UCP1 immunohistochemistry (F), semithin sections stained with toluidine blue (G), and transmission electron microscopy of adipocytes (H). Ip, lipid vesicle; mt, mitochondria; $\mathrm{nc}$, nucleus. Arrowheads show BeAT; arrow shows fusing lipid vesicles. Scale bars: $25 \mu \mathrm{m}$ (F and $\mathbf{G}) ; 5 \mu \mathrm{m}$ (H). (I) Mitochondrial content, TC content, adipocyte size, BeAT area in iAT, plasma glycerol levels, and lean weight on P10. (J) Transcription of BeAT hallmark genes in iAT on P10 of vehicle- or AKG-treated mice. ${ }^{*} P<0.05$; ${ }^{* *} P<0.01 ;{ }^{* *} P<0.001$, 1-way ANOVA with Dunnett's post hoc test (C) and Student's 2-tailed unpaired $t$ test (E, I, J). 
A

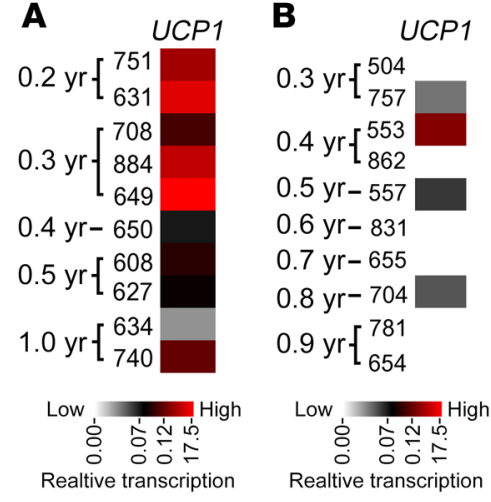

C

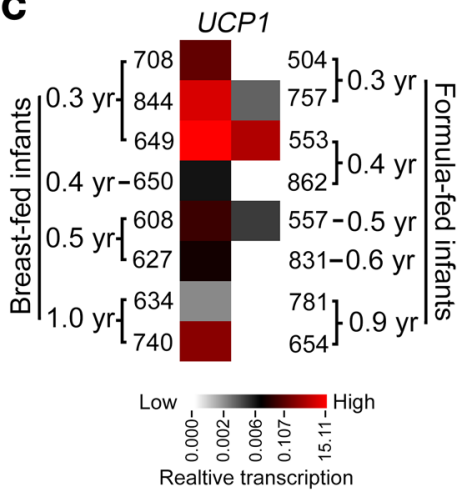

D Breast-fed infant Formula-fed infant

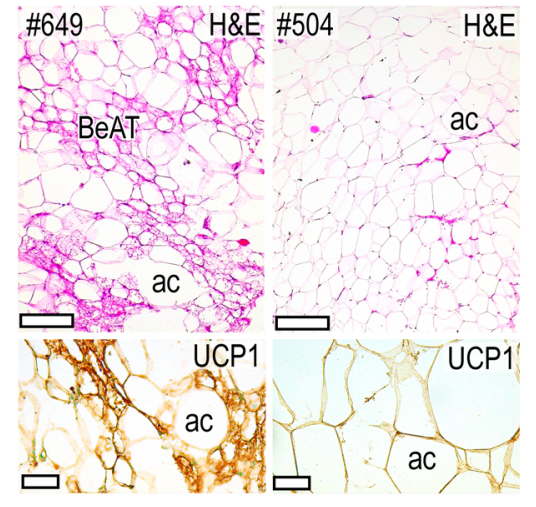

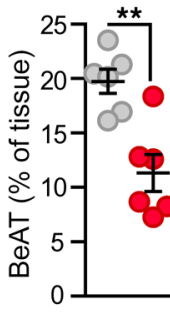

Breast-fed $0.3-0.6$ yr

- Formula-fed $0.3-0.6 \mathrm{yr}$

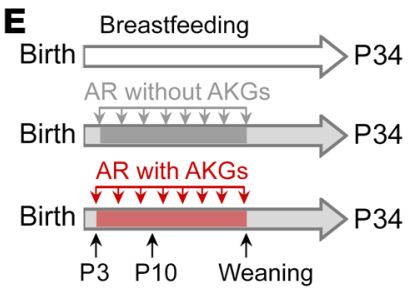

$\mathbf{F}$

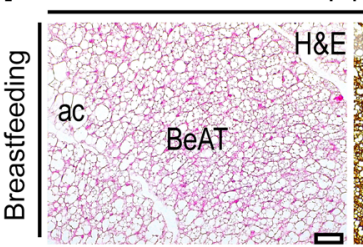

P10

P34
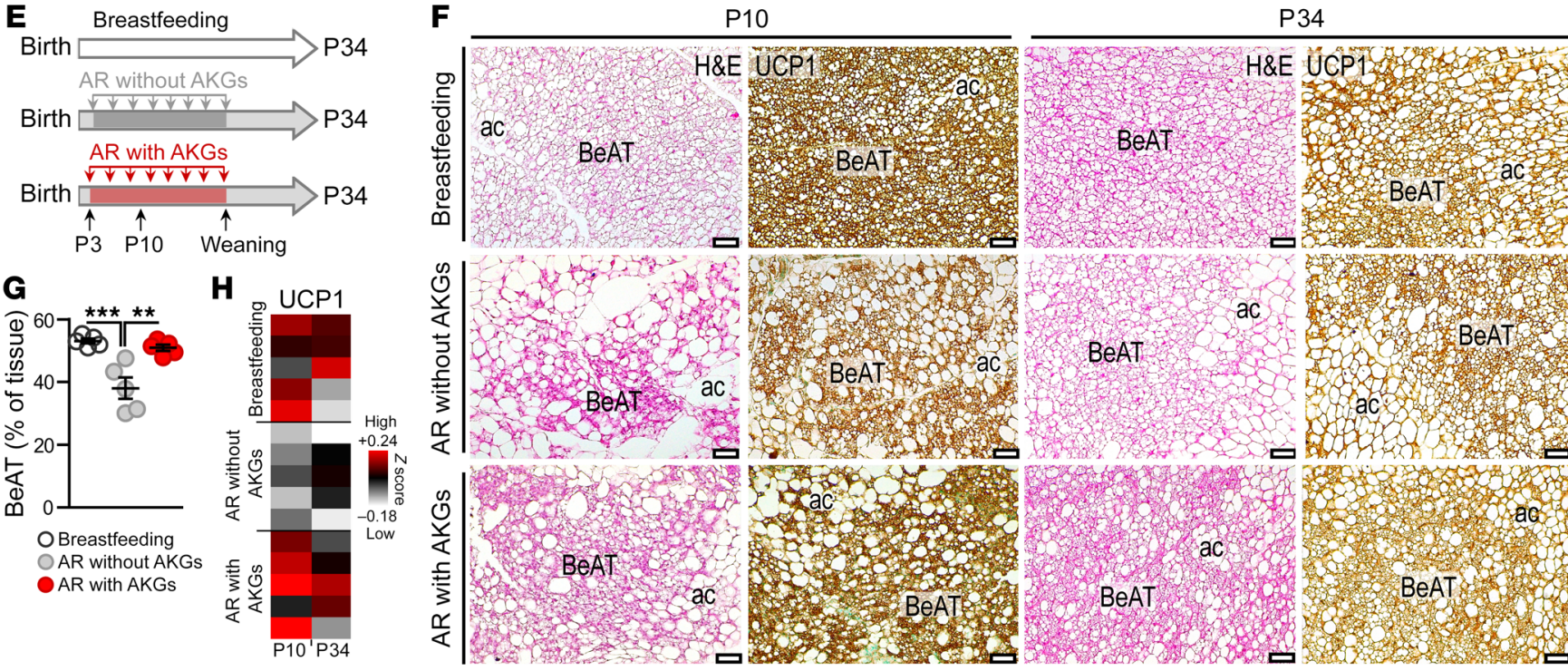

Figure 2. Lack of breastfeeding and lack of neonatal AKG intake reduce beige adipocyte content in the iAT. (A-C) Color-coded CIM showing the relative transcript level of UCP1 in human infant iAT. Also see Supplemental Figure 6A. (A) Breastfed infants, (B) formula-fed infants; age in years and the individual identifier number are indicated for each sample. (C) CIM showing relative UCP1 level in 0.3- to 1.0-yearold infants. UCP1 level was normalized to 3 reference genes (ACTB, TBP, HPRT1), and individual values were compared with the mean value of the 2 study groups. (D) Histology and BeAT content of the iAT in breastfed and formula-fed human infants. Scale bars: $100 \mu \mathrm{m}$ (H\&E); $25 \mu \mathrm{m}$ (UCP1). (E) Scheme of artificial rearing (AR) experiment. (F) Histology of the iAT at P10 and P34 in breastfed and AR mice. ac, white adipocyte. Scale bars: $80 \mu \mathrm{m}$. (G) BeAT content of the iAT at P34. (H) CIM showing relative UCP1 level in iAT on P10 and P34. ${ }^{* *} P<0.01$; ${ }^{* *} P<0.001$, Student's 2-tailed unpaired $t$ test (D); 1 -way ANOVA with Dunnett's post hoc test (G).

the natural mothers; the other 2 groups were artificially reared (AR) with formula milk and fostered by ovariectomized, hence nonlactating, mice (Figure 2E). One group of AR mice received formula milk lacking AKGs, while the other group received AKG-supplemented artificial milk. We used the CD1 mouse strain as it is amenable to AR studies and has the added advantage that the BeAT/WAT transition is slower than in C57BL/6 mice (Supplemental Figure 6C), allowing us to accurately determine whether AKG deficiency reduces BeAT content.

AR compromised BeAT content and UCP1 expression, and this was accompanied by an increase in the TG content of the iAT (Figure 2, F-H, Supplemental Figure 6D). When AKG levels of the artificial milk were restored to their physiological range (Figure 1B and Supplemental Figure 3E), BeAT content, UCP1 expression, and TG content in the iAT were similar to those in the breastfed mice (Figure 2, F-H, and Supplemental Figure 6D). No differences in body weight and nursing volume were noted between breastfed and AR groups along the study period (Supplemental Figure 6E). AR was without a notable effect on BAT development (Supplemental Figure 6, F and G). These findings show that deprivation from breast milk led to premature loss of BeAT, which was mitigated when AKGs were provided to the neonates.

AKGs induce a beige adipocyte phenotype in an ATM-dependent manner. To begin to understand the underlying mechanism by which AKGs maintain BeAT, we treated 3T3-L1 preadipocytes and adipocytes in vitro with AKGs, using concentrations that were comparable with the physiological levels in neonate plasma and iAT. Throughout the study, we used $100 \mathrm{nM} \mathrm{CA}, \mathrm{BA}$, or SA or an equimolar combination of the 3 AKGs for in vitro assays. AKG treatment failed to increase mitochondrial content or affect mitochondrial activity, glycerol release, or the transcription of BeAT-hallmark genes (Supplemental Figure 7, A-F, and Supplemental Figure 8A). Similarly, AKGs failed to affect BeAT gene transcription in iATderived preadipocytes (Supplemental Figure 8B). 
A

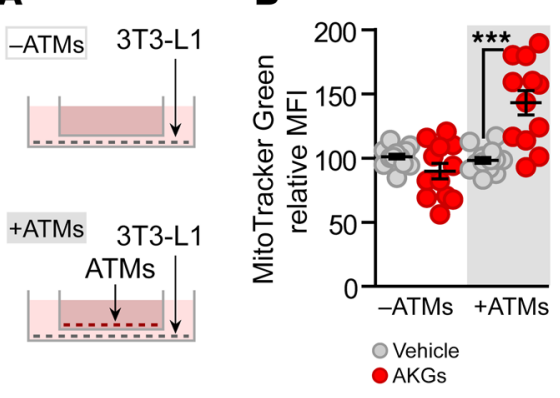

C MitoTracker Red

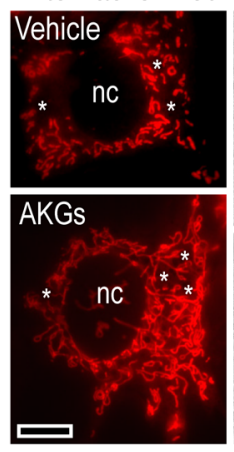

TEM

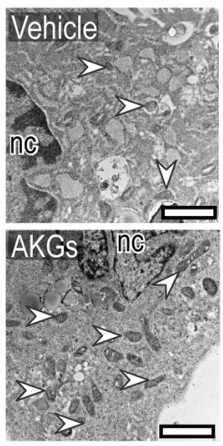

D

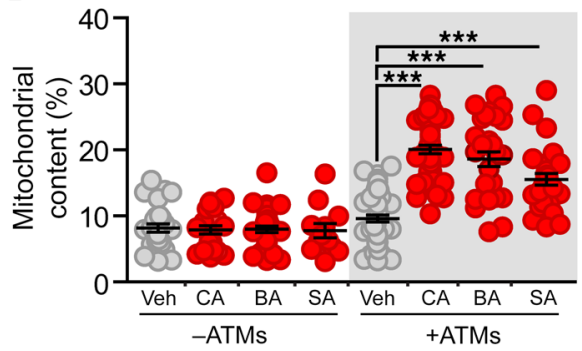

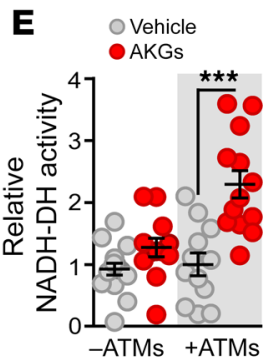
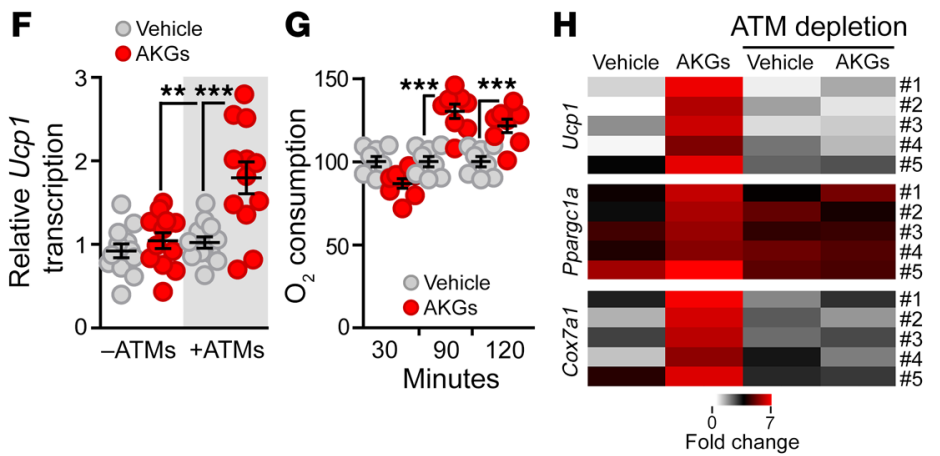

I

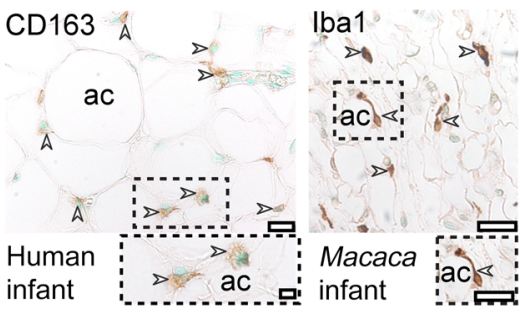

Figure 3. Macrophages are necessary for the effect of AKGs on adipocytes. (A) Schematic of a Transwell assay with ATMs and 3T3-L1 adipocytes. (B-G) Adipocytes were treated with vehicle or AKGs for 24 hours, without ATMs (-ATMs) or in the presence of ATMs (+ATMs). Each data point represents 1 Transwell assay (B, E-C) or 1 adipocyte (D). (B) MFI of MitoTracker Green in adipocytes after treatment. (C) MitoTracker Red staining and transmission electron microscopy (TEM) images of 3T3-L1 cells treated in the presence of ATMs. Asterisks show lipid droplets; arrowheads show mitochondria. Scale bars: $25 \mu \mathrm{m}$ (MitoTracker Red); 5 mm (TEM). (D) Mitochondrial content of 3T3-L1 cells, determined with image analysis. (E) Relative NADH-DH activity of adipocytes. (F) Ucp1 transcription in adipocytes. (C) Oxygen consumption of adipocytes treated with conditioned media of ATMs for 18 hours. ATMs were treated with vehicle or AKGs for 4 hours before cell culture media was collected and used for treating adipocytes. (H) CIM representation of BeAT-marker gene transcription in iAT of HFD-fed mice treated with vehicle or AKGs. ATMs were depleted with clodronate liposomes 24 hours before treatment in 1 group of mice ( $n=$ 5). (I) AT from human and M. mulatta infants, labeled for CD163 and lba1. Scale bars: $10 \mu \mathrm{m}$ (upper panels); $25 \mu \mathrm{m}$ (lower panels). ${ }^{* *} P<0.01 ;{ }^{* * *} P<0.001$, Student's 2-tailed unpaired $t$ test (B, E-G) 1-way ANOVA with Dunnett's post hoc test (D).

It has recently been shown that ATMs may induce BeAT development in adulthood through mechanisms that remain unclear and controversial (32-34). To determine whether ATMs play a role in the BeAT-inducing effect of AKGs, we cultured 3T3L1 adipocytes in the presence of ATMs using a Transwell system (Figure 3A). We plated adipocytes in the bottom chamber. The top chamber was left unseeded or was seeded with ATMs. In the presence of ATMs, AKG treatment increased adipocyte mitochondrial content (Figure 3, B-D, and Supplemental Figure 7G). MitoTracker Green and Red staining of mitochondria confirmed that they were functional (Figure 3, B-D, and Supplemental Figure $7 \mathrm{G}$ ), with a significantly increased relative mitochondrial NADH dehydrogenase (NADH-DH) activity (Figure 3E). The level of NADH-DH activity reflects mitochondrial respiration (35), which is increased in beige adipocytes (36). In the presence of ATMs, AKG treatment increased Ucp1 transcription in 3T3-L1 adipocytes (Figure 3F). J774A.1 macrophages had a similar, albeit less conspicuous, effect on adipocytes (Supplemental Figure 7H). Moreover, AKGs increased Ucp1 transcription and mitochondrial content in preadipocytes derived from adult mouse iAT in the presence of ATMs, and conditioned media of AKG-treated adult ATMs increased mitochondrial biogenesis in preadipocytes (Supplemental Figure 8, C-E). ATMs harvested from iAT of neonate mice had a similar effect on neonate preadipocytes (Supplemental Figure 8, F-H). Oxygen consumption of 3T3-L1 adipocytes was increased by conditioned media of AKG-treated ATMs (Figure $3 G)$. We labeled mitochondria with mito-thermo-yellow (MTY), a recently available fluorescent marker of mitochondrial thermogenesis and uncoupling (37-39). Reduced MTY signal indicated increased thermogenesis and uncoupling in adipocytes of AKG-treated neonate mice on P10 (Supplemental Figure 8I) and in both adult and neonate preadipocytes when they were treated with AKGs in the presence of adult or neonate ATMs, respectively (Supplemental Figure 8J).

Next we asked whether the absence of ATMs would impair AKG-induced transcription of BeAT-marker genes in vivo. We used high fat diet (HFD) feeding for 3 months to induce obesity, since it is known that ATM content is increased after HFD feeding in mice (40). We injected mice intraperitoneally with AKGs $(20 \mu \mathrm{mol} / \mathrm{kg}$ CA, $3.5 \mu \mathrm{mol} / \mathrm{kg} \mathrm{SA}, 40 \mu \mathrm{mol} / \mathrm{kg} \mathrm{SA}$ ) and found that Ucp1, Pparg$c 1 a$, and Cox7a1 were upregulated in adipocytes of the iAT within 4 hours (Figure 3H, $P<0.01$, Student's 2-tailed, unpaired $t$ test). Remarkably, when ATMs were depleted with clodronate liposomes 24 hours prior to AKG injection, AKG treatment failed to evoke these transcriptional changes (Figure $3 \mathrm{H}$ ). Overall, these findings suggest that ATMs are indispensable for the effect of AKGs. 
We previously showed that ATMs seed AT shortly after birth in mice (41). ATM accumulation has been shown to occur in childhood obesity (42). However, it is unknown whether human infant AT harbors resident ATMs under physiological conditions. Immunohistochemistry analysis revealed $\mathrm{CD} 163^{+} \mathrm{ATM}$ in human infant AT (Figure 3I and Supplemental Figure 9). CD163 is an early marker of tissue-resident macrophages, including ATMs (43). Similarly, ATMs in newborn Macaca mulatta expressed ionized calcium-binding adapter molecule 1 (Iba1) (Figure 3I and Supplemental Figure 9). Iba1 is an additional marker expressed by embryo-derived macrophages (44). These findings are especially important, since they demonstrate that ATMs are present when the physiologically relevant AT development of infancy occurs.

PAF mediates the effect of AKGs on BeAT development. To determine the effect of AKGs on ATMs, we performed a series of functional tests and treated them with $100 \mathrm{nM} \mathrm{AKGs} \mathrm{for} 18$ hours. AKGs had no effect on ATM viability, general morphology, or phagocytosis (Supplemental Figure 10, A-E). Using next-generation sequencing (NGS), we also assessed the transcriptional changes induced by AKGs. Transcripts related to ether lipid metabolism, especially to platelet-activating factor (PAF) precursor phosphocholine metabolites, were enriched in AKG-treated ATMs (Figure 4A). These observations prompted us to address whether AKGs had any effect on macrophage PAF production. Although AKG metabolism is poorly understood, recent studies have outlined the possibility that AKGs are utilized by a multienzyme-catalyzed process to generate PAF $(15,45)$, a potent phospholipid mediator $(26$, 27,46 ) (Figure $4 \mathrm{~B}$ ). In line with these predictions, we found that AKG treatment led to PAF release from mouse and human ATMs and that AKGs mirrored the transcriptional changes evoked by PAF in macrophages (Figure 4, C and D, and Supplemental Figure 10, F-J). Human THP-1 macrophages have been shown to convert AKGs to PAF (45), and we confirmed this production (Supplemental Figure 10I). The magnitude of AKG-induced PAF release was similar to that of the calcium ionophore A23187, a known trigger of PAF release (Supplemental Figure 10K). The transcriptional changes evoked by AKGs in macrophages were dependent on the PAF receptor (PTAFR), as they were abolished by its inhibition or ablation (Supplemental Figure 11, A and B). PAF is nonenzymatically converted into azelaoyl-PAF (AzPAF), which is a PPAR $\gamma$ agonist (47). Accordingly, the effect of AKGs on Arg1 and Il4ra transcription was mirrored by AzPAF and was dependent on PPAR $\gamma$ (Supplemental Figure 11, C-G).

AKG treatment failed to increase PAF release from preadipocytes and adipocytes (Figure 4C). However, ATMs were abundant in BeAT and were in close proximity to beige adipocytes (Figure 4, E and F, and Supplemental Figure 9), which may allow for paracrine effects by AKG-induced PAF release on adipocytes. Accordingly, depletion of ATMs in neonate mice compromised BeAT gene transcription in iAT, despite AKGs being provided by breastfeeding (Supplemental Figure 8K).

AKGs are structurally similar to 1-O-alkyl-2-acetyl-sn-glycerols (AAGs), which are substrates of de novo PAF synthesis, and to lyso-PAF, which is a substrate for PAF synthesis by phospholipid remodeling (Supplemental Figure 12). We evaluated whether genes encoding key enzymes of PAF synthesis from AAGs or lysoPAF were expressed by ATMs. We found that Chpt1, encoding choline phosphotransferase 1 (CHPT1, EC: 2.7.8.2), an enzyme that is responsible for de novo PAF synthesis from AAGs, was absent from mouse ATMs. However, it was robustly expressed by mouse adipocytes (Figure $4 G$ ). In contrast, Lpcat2, encoding lyso-PAF acetyltransferase 2 (LPCAT2, EC: 2.3.1.23), was mainly expressed by ATMs and was much less abundant in adipocytes in mouse (Figure 4G and Supplemental Figure 13A). LPCAT2 is known to remodel lyso-PAF into PAF (48). Protein expression of CHPT1 was confined to adipocytes in both mouse and human (Figure 4, G and H). LPCAT2 was detectable in mouse and human ATMs and to a lesser extent in adipocytes (Figure 4, $\mathrm{G}$ and H). PLA2G2A and PLA2G10 (phospholipase 2 group IIA and X, respectively, EC 3.1.1.4) can generate substrates for PAF remodeling. PLA2G2A was, however, not detectable in either ATMs or adipocytes in mouse (Supplemental Figure 13A), in accord with previous findings showing a frame-shift mutation in Pla2g2a in C57BL/6 mice (49). Human ATMs, but not adipocytes, however, expressed PLA2G2A (Supplemental Figure 13A). PLA2G10 was abundant in mouse adipocytes (Figure $4 G$ ), although human ATMs or adipocytes did not express it (Supplemental Figure 13A). PLA2G10 activity leads to the release of PAF, prostaglandin E2 (PGE2), and 15-deoxy-delta12,14-prostaglandin J2 (PGJ2) by the hydrolysis of membrane phospholipids (50-52). Prostaglandins are thought to induce BeAT development (53); however, AKG treatment failed to induce PGE2 or PGJ2 release from ATMs (Supplemental Figure 13B). These findings suggest that AKGs act specifically through PAF.

Next, we assessed the expression of PAF-catabolizing enzymes in ATMs and adipocytes. PLA2G7, also known as PAF acetylhydrolase (PAF-AH, EC 3.1.1.47) degrades PAF (28), and its expression was prominent in mouse and human ATMs (Figure 4, G and $\mathrm{H}$ ). In contrast, AGMO, which degrades AKGs and reduces the substrate pool for PAF synthesis (27), was expressed by adipocytes, while ATMs had only minor AGMO expression (Figure 4, G and H, and Supplemental Figure 4J). When we cultured mouse ATMs and adipocytes in vitro, we found a similar expression pattern: LPCAT2 and PLA2G7 were expressed by ATMs only, while PLA2G10 and AGMO were detectable in adipocytes only (Figure 4I). Of note, in vitro-cultured ATMs had much lower AGMO levels than freshly isolated ATMs (Supplemental Figure 13C). This suggests that AT-specific cues sustained AGMO level in ATMs.

Inhibition of LPCAT2 resulted in the absence of AKG-induced PAF release from ATMs (Figure 4J), whereas overexpression of LPCAT2 increased AKG-induced PAF release (Supplemental Figure 10, $\mathrm{L}$ and $\mathrm{M})$. Other inhibitors of PAF remodeling diminished, but did not abolish, PAF synthesis from AKGs (Figure 4J). In summary, these results show that ATMs, but not adipocytes, metabolize AKGs toward PAF (Figure 4K).

PTAFR is expressed in developing AT (Supplemental Figure 13D), where it has been shown to induce BeAT development (54). Additionally, Ucp 1 is directly upregulated by PAF in brown adipocytes (55). Accordingly, BeAT was absent from Ptafr/- mice, which also accumulated more TGs in the iAT than WT mice (Figure 5A and Supplemental Figure 13E). When we increased endogenous PAF synthesis in C57BL/6 mice by overexpressing human PLA2G2A (49), we found a robust increase in the BeAT content (Figure 5B and Supplemental Figure 13, F-H). 
A

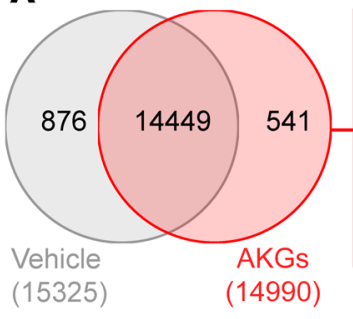

Gene ontology (GO 2017b, KEGG 2016, combined score ranking) starch and sucrose metabolism_hsa00500

fat digestion and absorption_hsa04975

negative regulation of macrophage activation GO:0043031

HMDB metabolites

Lyso-PC (HMDB11149)

PC-metabolites

Pla2g2a, Pla2g10
B

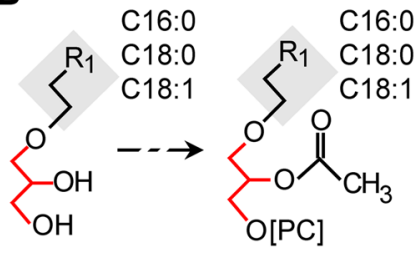

AKG Platelet activating factor (PAF)
C

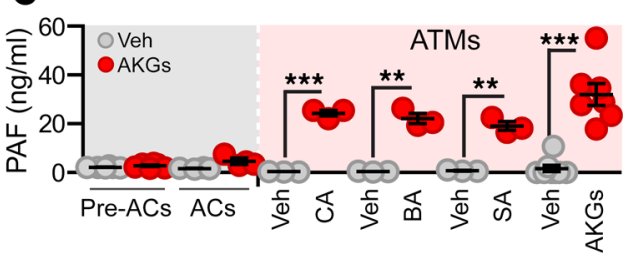

D

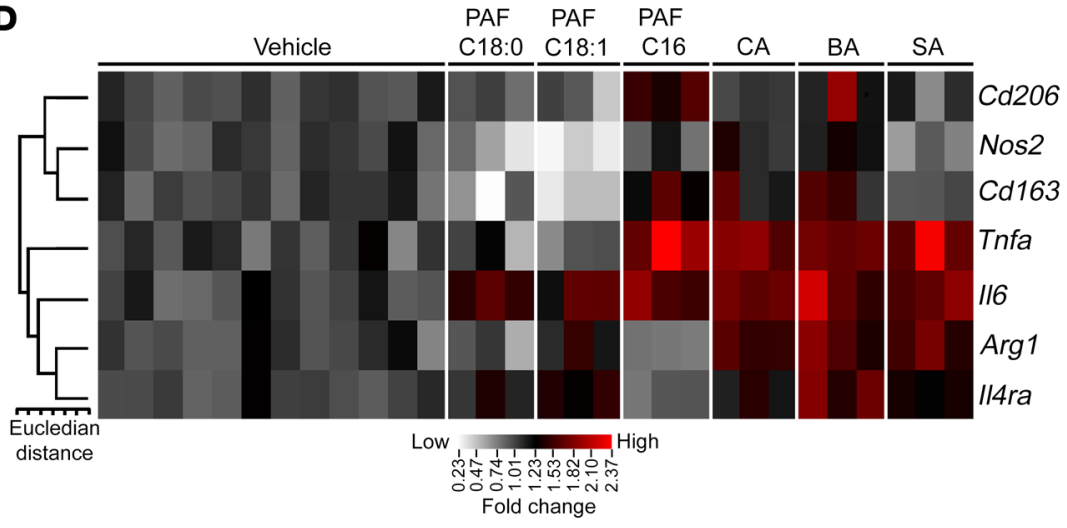

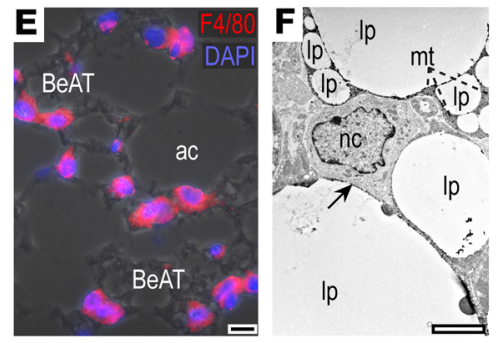

G

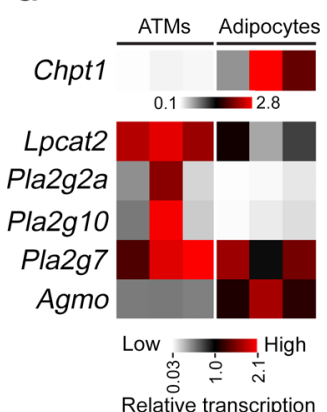

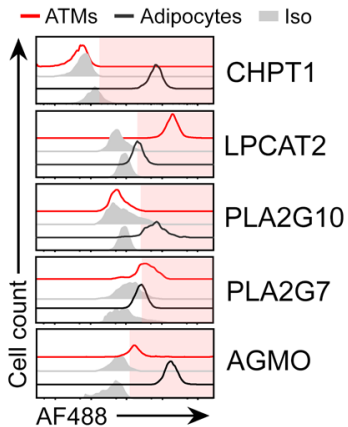

H

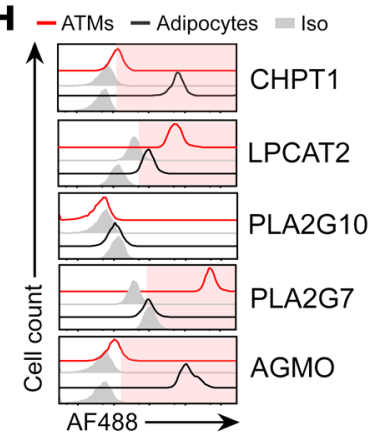

\section{I}
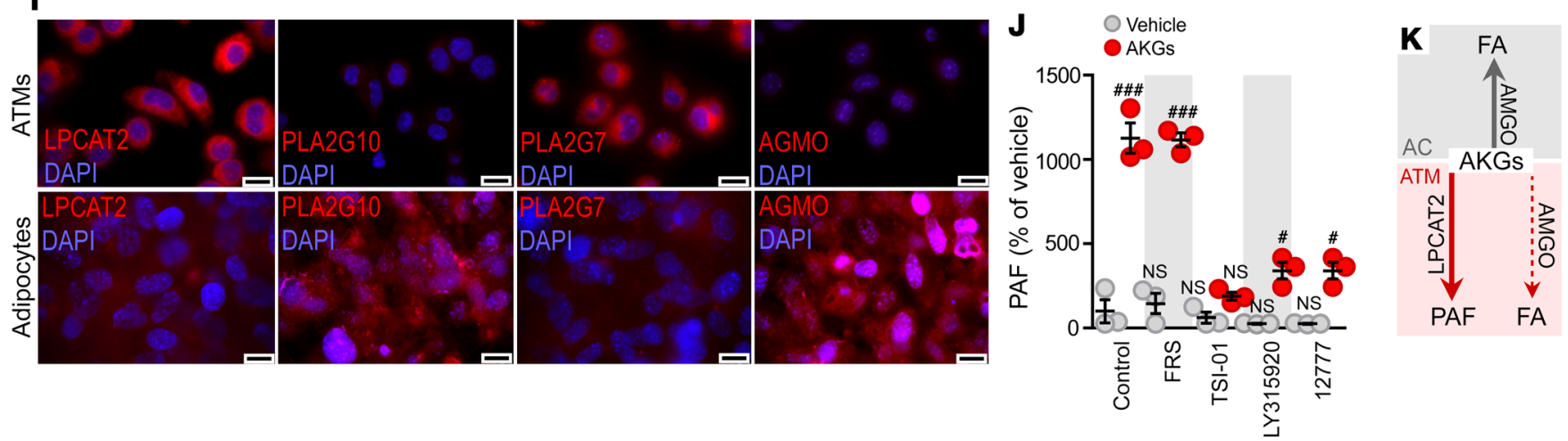

Figure 4. ATMs release PAF in response to AKGs. (A) NGS of vehicle- or AKG-treated macrophages. AKGs enriched transcripts related to phosphatidylcholine (PC) and lyso-PC metabolism. Pink bars, relative abundance of GO terms (combined score rankings). (B) AKGs can serve as backbones of the sn-acyl chain of PAF. (C) Top: PAF levels in cell culture media of 3T3-L1 pre-/adipocytes or ATMs treated with vehicle or AKGs for 30 minutes. Bottom: PAF release from 6-day-old neonate (P6) mouse preadipocytes (Pre-ACs), adipocytes (ACs), and ATMs in 30 minutes. (D) AKG-, and PAF-induced transcriptional changes in ATMs. (E) F4/80+ ATMs in mouse on P10. Scale bar: $10 \mu \mathrm{m}$. (F) TEM image of mouse BeAT on P10. Ip, lipid droplet. Scale bar: $4 \mu \mathrm{m}$. Arrow shows an ATM-like cell. (C) Transcript levels (left) and FACS analysis (right) of PAF-metabolizing enzymes in mouse ATMs and adipocytes. Iso, isotype control (H) FACS analysis of PAF-metabolizing enzymes in human ATMs and adipocytes. (I) Immunostaining of mouse ATMs and adipocytes cultured in vitro. Scale bars: $10 \mu \mathrm{m}$. (J) Effect of PAF synthesis inhibitors on AKG-induced PAF release in macrophages. FRS, farnesol (CHPT1 inhibitor); TSI-01, LPCAT2 inhibitor LY315920 and 12777, PLA2 inhibitors. ${ }^{*} P<0.01$; ${ }^{* * *} P<0.001$, Student's 2 -tailed unpaired $t$ test. ${ }^{*} P<0.05$; ${ }^{\# \#} P<0.001,1$-way ANOVA with Dunnett's post hoc test. (K) Schematic of AKG metabolism in adipocytes and ATM. 
A

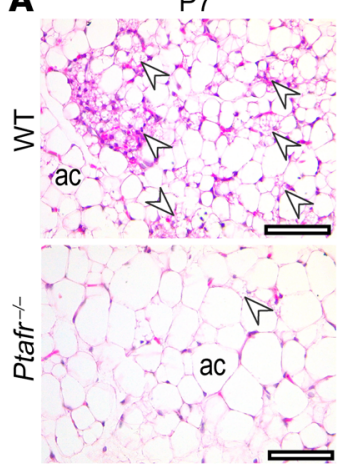

P10

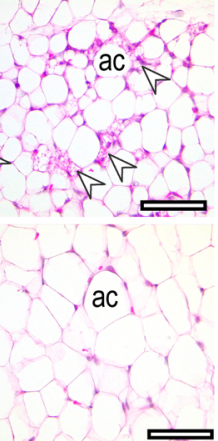

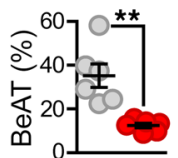

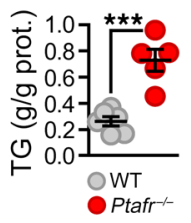

B

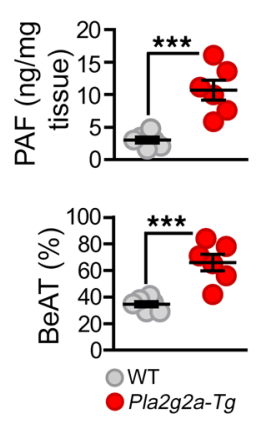

P7

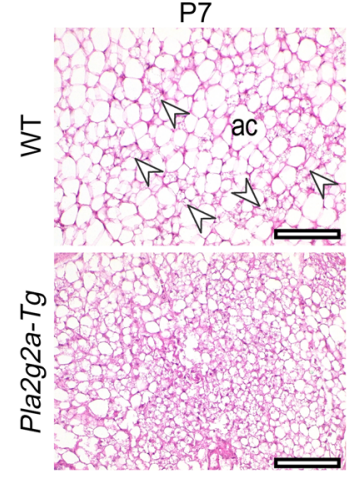

P10

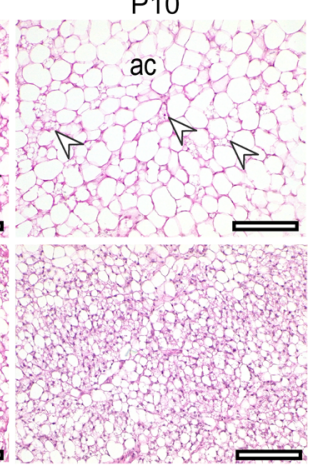

C

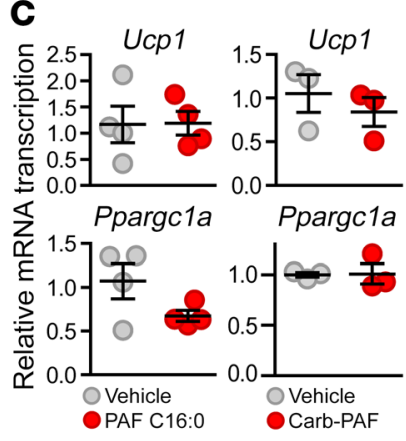

D

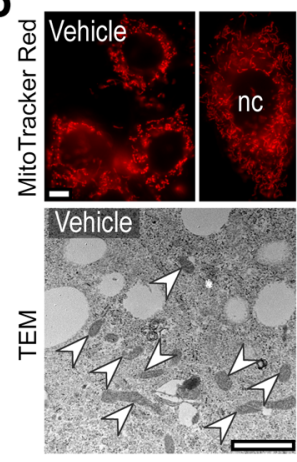

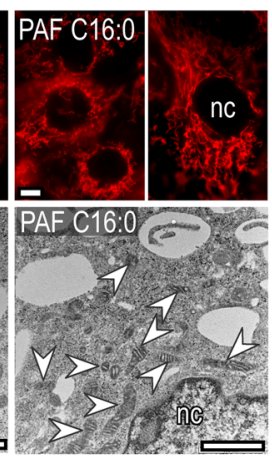

E

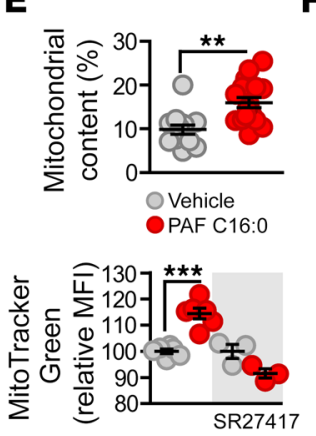

$\mathbf{F}$

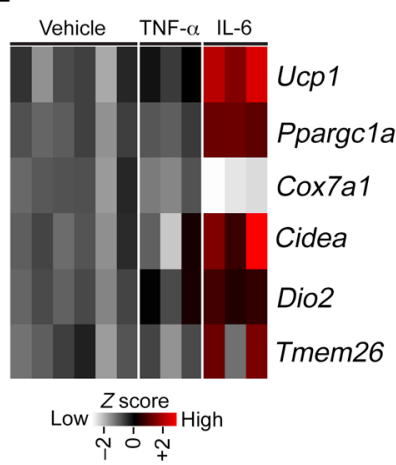

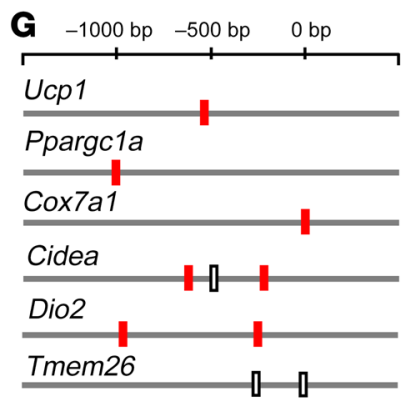

ISTAT3 \STAT

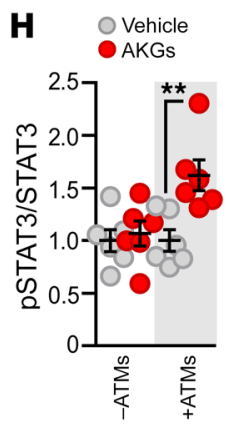

J

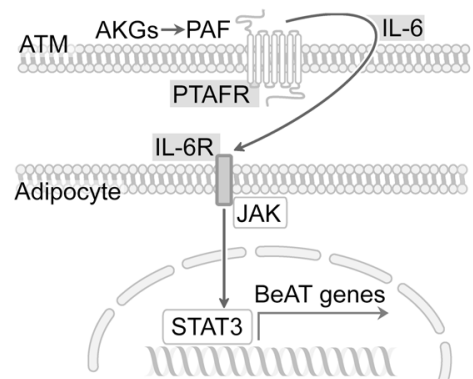

Figure 5. AKG-induced BeAT development is mediated by PAF/IL-6/STAT3 signaling. (A) Histology at P7 and P10; BeAT and TC content of AT at P10 in WT and Ptafr $^{-1-}$ mice. Arrowheads show BeAT. Scale bars: $65 \mu \mathrm{m}$. (B) PAF and BeAT content of AT at P10 and AT histology at P7 and P10 in WT and Pla2g2a-Tg (PLA2G2A-overexpressing) mice. Scale bars: $80 \mu \mathrm{m}$. (C) Relative transcription of Ucp1 and Ppargc1a in 3T3-L1 adipocytes treated with vehicle, $50 \mathrm{nM}$ PAF, or carbamyl PAF (Carb-PAF) for 18 hours. (D) MitoTracker Red staining and TEM image of 3T3-L1 adipocytes treated with conditioned media of ATMs for 18

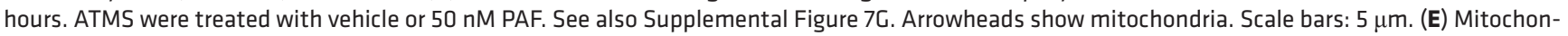
drial content and MFI of MitoTracker Green labeling of 3T3-L1 adipocytes treated with vehicle or $50 \mathrm{nM}$ PAF for 18 hours in the presence of ATMs. SR27417, PTAFR blocker. (F) Transcription of BeAT genes in adipocytes treated with $0.2 \mathrm{ng} / \mathrm{ml} \mathrm{IL-6}$ or $50 \mathrm{pg} / \mathrm{ml}$ TNF- $\alpha$ for 18 hours. (C) Transcription factor-binding site analysis of BeAT genes. (H) STAT3 phosphorylation in adipocytes treated with vehicle or AKGs for 30 minutes in Transwell assay shown in Figure $3 A$. (I) ATMs were treated with vehicle (Veh) or AKGs for 4 hours, and culture media were added to adipocytes for a further 18 hours. CIM summarizes transcriptional changes evoked in adipocytes. STAT3 was inhibited with $500 \mathrm{nM}$ cucurbitacin I, JAK/STAT3 with $280 \mathrm{nM}$ ruxolitinib. (J) Schematic of the mechanism by which AKGs trigger BeAT gene transcription in adipocytes. ${ }^{* *} P<0.01 ;{ }^{* *} P<0.001$, Student's 2-tailed unpaired $t$ test.

Given that these data suggest that PAF release from AKGs may be a trigger for BeAT development, we next addressed the potential underlying signal mechanisms. PAF induces $\mathrm{p} 38$-MAPK phosphorylation (56), which is required for Ppargcla and Ucp1 transcription (57). However, conditioned media of AKG-treated ATMs failed to induce p38-MAPK phosphorylation in adipocytes (Supplemental Figure 13I). More surprisingly, PAF treatment of 3T3-L1 adipocytes failed to induce the transcription of BeAT-marker genes or increase mitochondrial load (Figure 5C and Supplemental Figure 13I). In the presence of ATMs, however, PAF increased the mitochondrial load of adipocytes (Figure 5, D and E), an effect that was diminished when PTAFR was pharmacologically inhibited (Figure 5E). AKGs induced IL-6 release from ATMs (Supplemental Figure 13J), which effect was mediated by PAF/PTAFR (Supplemental Figure 11A). When we treated adipocytes with $0.2 \mathrm{ng} / \mathrm{ml}$ IL-6 - mimicking AKG-induced IL-6 release from ATMs - the transcription of 
A

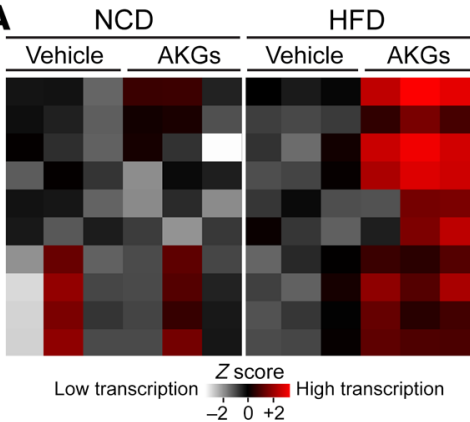

B

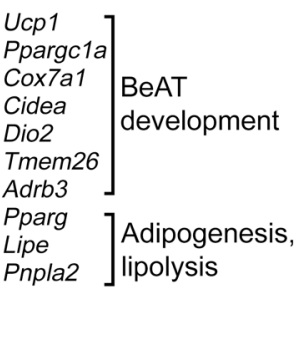

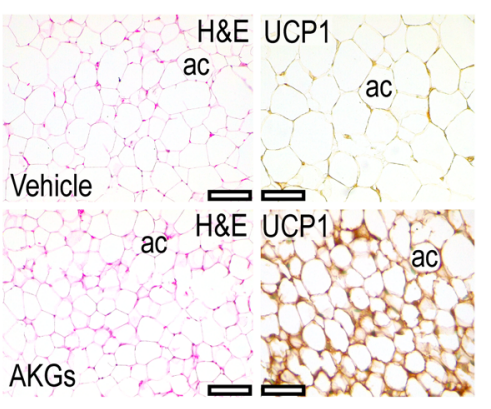

C Lepr $r^{\text {dbldb }}$

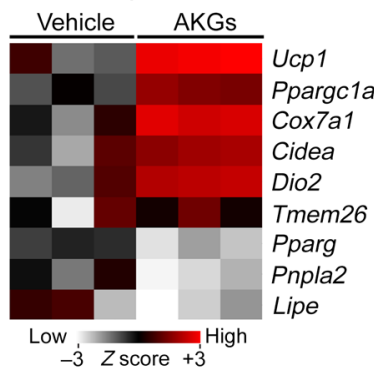

D
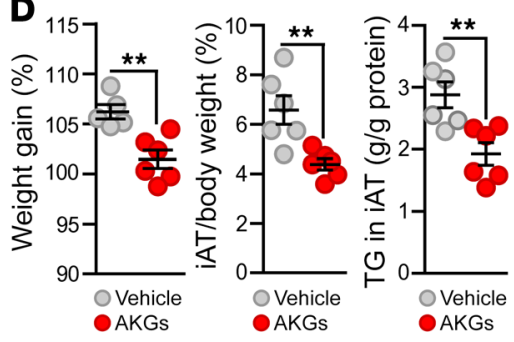

E

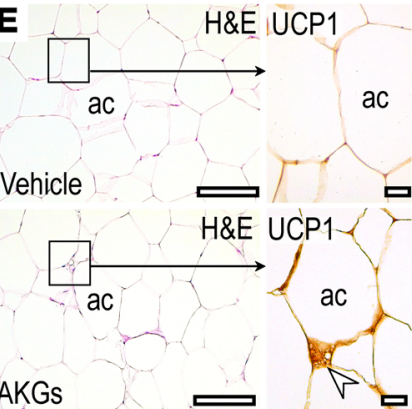

AKGs
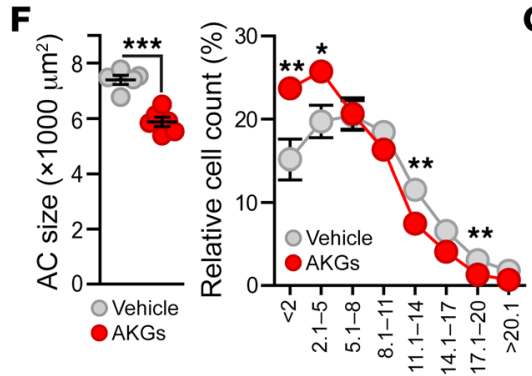

G

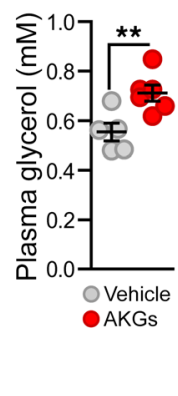

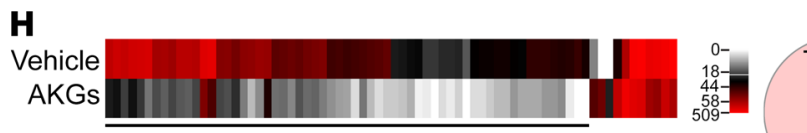

Gene ontology of AKG-suppressed transcripts Focal adhesion Mus musculus WP85

Inflammatory response pathway Mus musculus WP458

Focal adhesion-PI3K-Akt-mTOR-signaling pathway Mus musculus WP2841

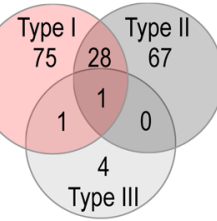

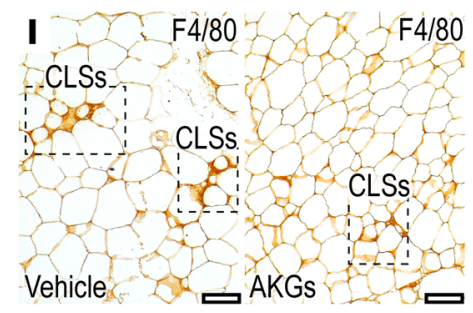

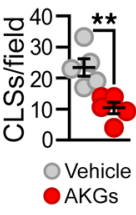

Figure 6. Effects of dietary AKG supplementation in obesity. (A) Transcript levels of BeAT genes and genes associated with adipogenesis and lipolysis in IAT of lean, and HFD-fed mice, treated with vehicle or AKGs. (B) Histology and UCP1 immunohistochemistry of iAT of HFD-fed mice treated with vehicle or AKGs. Scale bars: $50 \mu \mathrm{m}$. (C) Transcript levels of BeAT genes and genes associated with adipogenesis and lipolysis in iAT of $L e p{ }^{d b / d b}$ mice treated with vehicle or AKGs. (D) Weight gain within 1 week, iAT weight, and TC content of iAT of Leprdb/db mice treated with vehicle or AKCs. (E) Histology, (F) adipocyte size, and (G) plasma glycerol level in Leprdb/ab mice treated with vehicle or AKGs. Scale bars: $50 \mu \mathrm{m}$ (H\&E); $10 \mu \mathrm{m}$ (UCP1). Arrowheads show UCP1+ adipocyte. (H) NGS analysis of vehicle- and AKG-treated ATMs, showing relative abundance of transcripts. Pink bars, combined score ranking of GO terms. Venn diagram of AKG-suppressed interferon-responsive genes, clustered according to the type of interferon response. (I) Crown-like structures (CLSs) in iAT of Lepr $^{d b / d b}$ mice treated with vehicle or AKGs. Scale bars: $50 \mu \mathrm{m}$. Quantification of CLSs in iAT of Lepr ${ }^{d b / d b}$ mice treated with vehicle or AKGs. ${ }^{*} P<0.05$; ${ }^{* *} P<0.01 ;{ }^{* * *} P<0.001$, Student's 2-tailed unpaired $t$ test.

BeAT genes (Ucp1, Ppargc1a, Cidea, Dio2, Tmem26) was markedly upregulated (Figure 5F and Supplemental Figure 8L). Cox7a1, which has been recently found dispensable for BeAT (58), was not upregulated by IL-6 (Figure 5F). AKGs caused a moderate TNF- $\alpha$ secretion from ATMs (Supplemental Figure 13J), which, however, did not affect BeAT gene transcription (Figure $5 \mathrm{~F}$ ).

IL-6 can act through STAT3 (36). Ucp1, Ppargcla, Cidea, Cox7a1, Dio2, and Tmem26 have binding sites for STAT3 and STATs upstream of their promoters (Figure 5G). AKGs induced STAT3 phosphorylation in 3T3-L1 adipocytes, when ATMs were present (Figure 5H). Conditioned media from AKG-treated ATMs induced BeAT gene transcription in adipocytes, which effect was abolished by STAT3 or JAK/STAT3 inhibition (Figure 5I). Together, our data show that PAF lacks a direct BeAT-inducing effect. However, by acting through an autocrine loop, it increases IL-6 release from ATMs, which ultimately evokes beige adipocyte differentiation (Figure 5, I and J).
AKGs do not affect adult lean AT, but induce BeAT development in obese $A T$. We next tested to determine whether AKG supplementation could prolong the existence of BeAT after breastfeeding is discontinued. To do this, we fed lean 10-week-old male C57BL/6 mice with an AKG-rich diet $(16 \mu \mathrm{mol} / \mathrm{kg} / \mathrm{d} \mathrm{CA}, 2.6 \mu \mathrm{mol} / \mathrm{kg} / \mathrm{d} \mathrm{BA}$, $30 \mu \mathrm{mol} / \mathrm{kg} / \mathrm{d} \mathrm{SA}$ ) for 1 month. Contrary to our expectation, transcription of BeAT-associated genes remained unaffected in the iAT (Figure 6A). This unexpected result prompted us to ask whether AKGs induce BeAT development in obesity. We fed C57BL/6 mice a HFD for 3 months and then treated them with vehicle or with AKGs $(16 \mu \mathrm{mol} / \mathrm{kg} / \mathrm{d} \mathrm{CA}, 2.6 \mu \mathrm{mol} / \mathrm{kg} / \mathrm{d} \mathrm{BA}, 30 \mu \mathrm{mol} / \mathrm{kg} / \mathrm{d} \mathrm{SA})$ for an additional month. Subsequently, AKG treatment upregulated BeAT genes in iAT, including Ucp1, Ppargc1a, Cox7a1, Cidea, Dio2, and Tmem 26 (Figure 6A and Supplemental Figure 14A). We also detected a moderate increase in the transcription of Adrb3 and its downstream targets Lipe and Pnpla2. Furthermore, iAT mass and adipocyte size were reduced (Supplemental Figure 14, B and C) and 
A

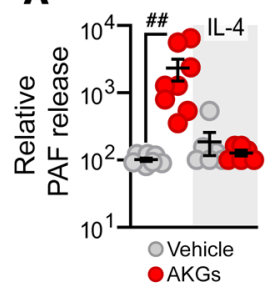

D

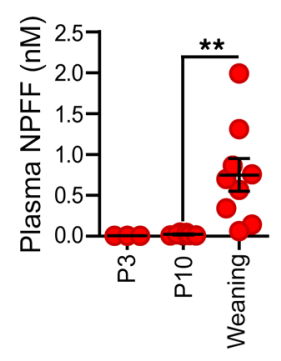

B

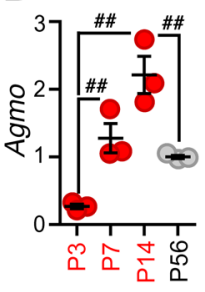

C
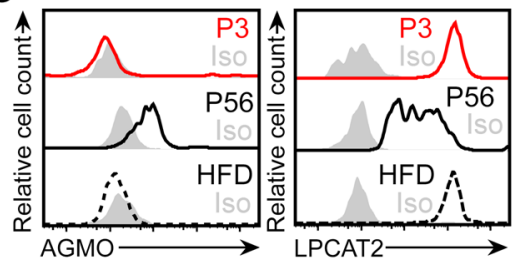

E

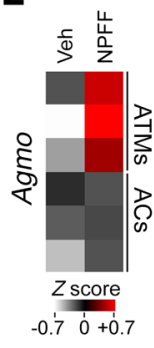

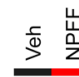

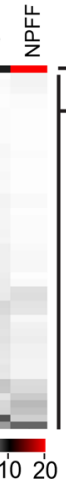

-Park2

Lysosome (GO:0005764) - Lysosomal lumen (GO:0043202) Vacuolar lumen (GO:0005775) Lytic vacuole (GO:0000323)

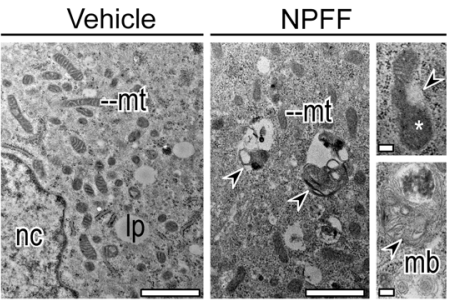

$\mathbf{F}$

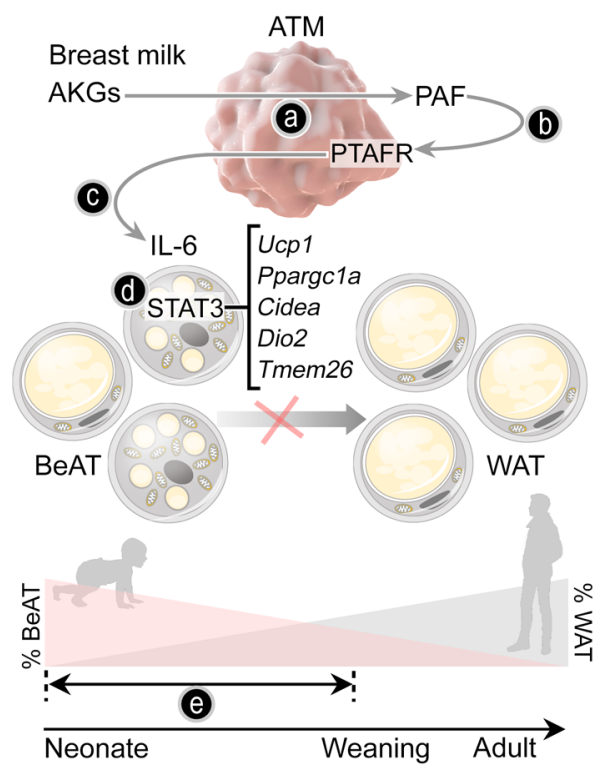

Figure 7. AKG-mediated signaling to adipocytes is inactivated at BeAT/WAT transition. (A) ATMs were treated with vehicle or AKCs for 30 minutes, and PAF levels were measured in the cell culture media. ATMs were pretreated with vehicle or $10 \mathrm{ng} / \mathrm{ml}$ IL-4 for 18 hours. (B) Transcript levels of Agmo in mouse ATMs isolated at P3, P7, P14, and P56. One data point represents pooled ATM samples from 9 to 11 neonate (P3, P7, P14) and 3 to 4 adult (P56) mice. ${ }^{\#} P<0.01$, 1-way ANOVA with Dunnett's post hoc test. (C) FACS histogram showing ATM expression of ACMO and LPCAT2 at P3 and P56 and following 3-months HFD feeding in mouse. (D) Plasma NPFF levels in C57/BL6 mouse at P3 and P10 and at weaning. ${ }^{* *} P<0.01$, Student's 2-tailed unpaired $t$ test. (E) Left: effect of NPFF on Agmo transcription in ATMs and 3T3-L1 adipocytes. Cells were treated with vehicle or $1 \mathrm{nM}$ NPFF for 18 hours in vitro. Another data set of NPFF-induced transcriptional changes in macrophages has been published previously (62) (GEO GSE90658). Right: NGS analysis of 3T3-L1 adipocytes treated with vehicle or NPFF for 18 hours. Ontology of significantly upregulated transcripts $(P<0.001)$ is shown. Pink bars, combined score ranking; gray bars, rank-based ranking of GO terms. TEM images of 3T3-L1 adipocytes treated with vehicle or $1 \mathrm{nM}$ NPFF for 18 hours. Arrowheads show mitophagosome membranes. mb, multilamellar body. Scale bars: $5 \mu \mathrm{m} ; 1 \mu \mathrm{m}$ (insets). (F) Schematic of AKG-mediated signaling in AT. (a) Breast milk AKGs are metabolized by ATMs into PAF. (b) In an autocrine loop, PAF triggers IL-6 release from ATMs. (c) The secreted IL-6 activates adipocyte JAK/STAT3 signaling. (d) This triggers BeAT gene transcription and prevents a premature BeAT/WAT transition. (e) Under physiological conditions, AKG signaling in AT is active only in infancy and is inactivated in lean adult AT.

$\mathrm{UCP}^{+}$adipocytes were abundant within the iAT of the AKG-treated mice (Figure 6B). The effect of AKGs in epididymal AT (eAT) and BAT was not apparent (Supplemental Figure 14D). Similarly, when we treated leptin-resistant diabetic obese Lepr ${ }^{d b / d b}$ mice with AKGs $(16 \mu \mathrm{mol} / \mathrm{kg} / \mathrm{d} \mathrm{CA}, 2.6 \mu \mathrm{mol} / \mathrm{kg} / \mathrm{d}$ BA, $30 \mu \mathrm{mol} / \mathrm{kg} / \mathrm{d} \mathrm{SA})$ for 1 month, the transcription of BeAT genes was strongly upregulated in the iAT (Figure 6C and Supplemental Figure 14A). Moreover, weight gain, iAT weight, and TG content were all reduced in these mice (Figure 6, D-F) without a prominent effect on eAT (Supplemental Figure 14, E and F). BeAT development is inhibited by leptin resistance (59); however, we detected an increase in mitochondrial DNA content of iAT after AKG treatment (Supplemental Figure $14 \mathrm{G}$ ), and $\mathrm{UCP1}^{+}$adipocytes were detectable in the iAT of AKG-treated mice (Figure 6E) along with a reduced adipocyte size (Figure 6F) and increased plasma glycerol level (Figure 6G).

NGS analysis showed that AKG treatment suppressed the transcription of interferon-inducible genes in ATMs (Figure 6H). The effect of PAF was detectable by specific changes in the transcription of macrophage activation genes; however AKGs did not polarize macrophages (Supplemental Figure 11, C-G). AKG treatment also reduced the amount of crown-like structures (Figure 6I), which are sites of inflammatory ATM activation (60-62). Indirect calorimetry indicated that AKGs decreased the respiratory exchange rate (RER) (Supplemental Figure 14H). This decrease of RER, together with a marked decrease in iAT, reduced adipocyte size, and increased BeAT gene transcription (Figure 6, A and B, and Supplemental Figure 14, A-C), indicates an increased oxidation of lipids in response to AKGs. AKGs did not affect metabolic rate and energy expenditure substantially (Supplemental Figure 14H), which suggests that loss of fat mass was not a result of increased activity. In summary, AKG treatment induced BeAT and reduced iAT mass in obese mice. However, AKGs remained ineffective in lean adult animals.

AKG-induced BeAT development is inactivated in adulthood. BeAT development is associated with increased mitochondrial content and uncoupling, which can have unfavorable outcomes when it occurs in excess $(63,64)$. The expansion of the mitochondrial network is thus maintained in a physiological range by signals that remain the subject of intense research $(65,66)$. It is intriguing that AKGs had an effect only in neonates and in adult obese mice and were ineffective in lean adult mice. Thus, we searched for a mechanism that could inactivate AKG signaling in the adult, lean state. The polarization state of ATMs is known to be different in lean and obese AT (67). Lean AT harbors ATMs, which have similarities to proresolving, so-called M2 macrophages (40). Conversely, obesity evokes a specific activation state of ATMs that 
resembles features of inflammatory, so-called M1 macrophages $(40,67)$. Because ATM activation has an impact on AT function, we asked whether it could affect the responsiveness of ATMs to AKGs. We induced M1 and M2 polarization in vitro and found that transcription of Lpcat 2 and Pla2g10, which are encoding enzymes involved in AKG/PAF conversion and PAF remodeling, respectively, was increased by M1 polarization (Supplemental Figure 15, $\mathrm{A}$ and $\mathrm{B})$. In contrast, M2 activation reduced their transcription, increased PAF elimination to lyso-PAF (Supplemental Figure 15, A and B), and abolished AKG conversion to PAF (Figure 7A). It is known that Agmo transcription is diminished by M1-activating stimuli $(26,62)$ (Supplemental Figure 15, C and D), whereas M2 polarizing signals increase Agmo transcription in ATMs (62). Accordingly, we found that IL-4/STAT6 signaling controlled Agmo transcription and AGMO was expressed and localized to intracellular membranes in IL-4-treated macrophages (Supplemental Figure 15, E and F), which serve as the site of AKG accumulation and PAF synthesis $(24,28,68,69)$.

AGMO effectively reduces the substrate pool for PAF synthesis $(26,27)$ and thus has the potential to diminish the effects of AKGs. Accordingly, when we overexpressed Agmo in J774A.1 macrophages, the AKG-induced PAF release, as well as the AKG-induced Tnfa and Il6 expression, was abolished (Supplemental Figure $15 \mathrm{G}$ ). We also found a striking difference in the expression of AGMO between neonate and adult ATMs. ATM expression of Agmo increased progressively in neonate mice, reaching a peak at $\mathrm{P} 14$ (Figure 7B). In neonate mice $\mathrm{AGMO}^{+} \mathrm{ATMs}$ were scarce (Figure 7C) and only self-renewing or IL-4-treated ATMs had significant AGMO expression (Supplemental Figure 15H). $\mathrm{AGMO}^{+} \mathrm{ATMs}$ were detectable in adults, and in contrast, $\mathrm{AGMO}^{+} \mathrm{ATMs}$ were undetectable in obese mice, which was similar to what occurred in neonates (Figure 7C). In contrast, LPCAT2 was steadily expressed by ATMs throughout life (Figure 7C).

We have recently shown that ATM-specific expression of AGMO is increased by neuropeptide FF (NPFF), which is an endocrine signal for homeostatic maintenance of the ATM pool and which increases the enrichment of M2-like ATMs in adult AT (62). NPFF increases AGMO expression through NPFF receptor 2 (NPFFR2) by sustaining IL-4/STAT6 signaling (62). NPFFR2 is the main receptor for NPFF in $\mathrm{AT}(70,71)$, and its expression is restricted to ATMs (62). We found that the plasma concentration of NPFF in neonate mice was negligible and increased at weaning (Figure 7D). The similarity in the timing of changes in plasma NPFF levels and BeAT/WAT transition suggests that activation of NPFF/NPFFR2 signaling at weaning is one signal that increases AGMO expression in ATMs (Figure 7E) and eventually inactivates AKG effects.

Finally, we asked whether NPFF could have an ATM-independent effect on BeAT/WAT transition. We treated 3T3-L1 adipocytes with NPFF in vitro and measured changes in gene transcription by NGS. We found that the effect of NPFF on adipocytes was strikingly different from its effect on ATMs: whereas NPFF sustained STAT6 signaling and inhibited various inflammatory pathways in ATMs (62), in adipocytes, it induced the prominent expression of Park2 in addition to lysosome-associated transcripts (Figure 7E). Park2 encodes parkin, a ubiquitin ligase that has recently been shown to allow BeAT/WAT transition by increas- ing mitophagy (65). Accordingly, NPFF-treated 3T3-L1 adipocytes contained a greater number of mitophagosomes than vehicle-treated adipocytes (Figure 7E). The adipocyte effect of NPFF was due to its binding to NPFFR1, which was expressed by 3T3-L1 adipocytes (Supplemental Figure 15I). Autophagy allows metabolic adaptation (72), and it coordinates with mitochondrial biogenesis to maintain healthy metabolism $(65,66,73)$.

Our study shows that breastfeeding provides AKGs for neonate ATMs, which in turn sustain BeAT (Figure 7F). The transition from neonate type BeAT to adult-type WAT is associated with the inactivation of AKG signaling, at least in part due to the M2-like polarization of ATMs in the lean AT. In infancy, AKG signaling is critical for protection from obesity, since a premature loss of BeAT can cause WAT excess $(3,20)$ (Figure 7F).

\section{Discussion}

Lipid signaling is essential for AT physiology $(1,11)$ and also dictates the metabolic outcome of ATM functions $(1,11,74)$. Breast milk, the most natural nutrient for infants, is a rich source of lipid mediators $(7,10)$. For instance, breast milk lipids support the infant's immune functions (14-16), and alterations in breast milk lipids can cause chronic inflammatory diseases in the infant $(8,16$, 17). Most of the breast milk lipids are also provided in the adult diet (10); hence, their effect may not be specific for infancy. Here we show that AKGs are breast milk-specific signals, which protect from obesity and maintain BeAT in infants. To our knowledge, this is the first identified maternal lipid signal mechanism that has a direct impact on BeAT development.

AKGs are metabolized by ATMs, which in turn facilitates the local release of IL- 6 in the vicinity of adipocytes and induces their differentiation into BeAT through JAK/STAT3. This ATMadipocyte signaling is active only in early postnatal life, when BeAT makes up the majority of the developing AT. AKG signaling is hence active during a sensitive period of AT development, and this determines obesity status in later life (3).

Local PAF production by ATMs is the first element of AKG signaling. Macrophage-derived PAF is central to host defense $(15,75,76)$. In the context of AT health, PAF was hence initially considered as a metabolically nonfavorable mediator, since it can trigger AT inflammation (48). Supporting this scenario, the lack of PTAFR protects mice from diet-induced AT inflammation (55). However, the absence of PTAFR also increases lipogenesis (55), and PTAFR-deficient mice develop age-related obesity and are more susceptible to diet-induced obesity than WT mice $(54,77$, 78). Conversely, mice overexpressing human PLA2G2A, a major enzyme of PAF remodeling, are protected from diet-induced obesity and present increased thermogenesis (49). BAT function is also impaired in PTAFR-deficient mice, due to the lack of PAFinduced upregulation of Ucp1 mRNA in brown adipocytes (54, 77). It has also been shown that the plasma level of PLA2G7 positively correlates with the degree of abdominal obesity in children (79). Since PLA2G7 eliminates PAF (80) and obese children have compromised BeAT content (20), it is possible that PAF signaling impedes obesity development in children. This would be consistent with our findings showing a function for PAF/PTAFR signaling in maintaining BeAT. Reinforcing this notion, ablation of PTAFR led to the absence of BeAT, whereas increasing the levels 
of PAF via PLA2G2A overexpression resulted in an overwhelming BeAT content in neonate mice $(49,50)$.

We found that IL- 6 was the downstream mediator of PAF, which activates the transcriptional program of BeAT development through STAT3. ATM-derived IL-6 has long been considered a deleterious cytokine, which together with other inflammatory mediators triggers systemic metabolic disease. However, mice with global loss of IL-6 develop insulin resistance (81) and are more prone to diet-induced obesity (82). IL-6 activates mitochondrial biogenesis in AT (82), increases pancreatic insulin secretion (83), and plays a role in the hypothalamic control of appetite (84). It has also been shown that AT development and remodeling are critically dependent on inflammatory signaling early in postnatal life (85). Most recently, $\beta_{3}$ adrenergic stimulation of lipolysis has been shown to trigger IL-6/JAK/STAT3 signaling and promote beige adipocyte development (36). Our findings on the role of AKG/ PAF/IL-6/STAT3 signaling in BeAT development are in line with these recent findings and confirm that BeAT development in the infant requires transient inflammatory signaling (86). In adult AT, M2 polarization of ATMs has been shown to increase BeAT content $(32,87)$, although the necessity of M2-activated ATMs for WAT/ BeAT transformation has been recently challenged (33). Since M2 polarization suppresses the ability of ATMs to synthesize PAF from AKGs, it suggests that M2 ATMs play a role in BeAT differentiation in cold-stressed adult AT (88), rather than in the developing neonate AT. These findings expand the known metabolic role of inflammatory ATMs (11). Moreover, ATM number is decreased in lean young adults (89), and the majority of ATMs adopt an M2-like activation state in the adult lean AT (11). The low ATM number and the prevalence of M2 activation can impede the effect of AKGs in lean adults and limit the physiological impact of AKGs on infancy.

The reason that AKGs are specifically enriched in the breast milk is explained by the lipid secretion mechanism of the mammary gland cells. Lipid vesicles are released together with the apical cell membrane by so-called apocrine secretion (10). AKGs are enriched in the apical cell membrane of the lactating gland cells (24) and synthesized within the cell membranes due to remodeling of other ether lipids $(24,28,68,69)$; hence, apocrine secretion allows AKG enrichment in the milk. Cow milk contains negligible AKGs, which is at odds with the finding of AKG synthesis and release in cow colostrum and milk (68). This may be due to an increased catabolism of AKGs in cow milk (24). Moreover, the amount of milk produced daily by a dairy cow is magnitudes higher than of the other species tested and may lead to the dilution of AKG concentration to a negligible level. Importantly, in bovine neonates, the subcutaneous AT is free from BeAT, whereas other mammalian species have BeAT during the period of breastfeeding (90). This also suggests that breast milk AKGs are direct signals of BeAT development and that the lack of AKGs is mirrored by the lack of BeAT.

We observed a marked decrease in BeAT content and UCP1 transcription in the inguinal subcutaneous fat compartment in human infants when breastfeeding was limited or absent. BeAT has previously been reported in subcutaneous and visceral fat depots in children (20). Supraclavicular, cervical, and interscapular regions also contain BeAT-rich fat depots (91), which are often considered to be BAT $(21,92)$. Because these fat depots have a metabolic role in adolescence (92), it is intriguing to question whether development of these fat depots is affected by breastfeeding. Surgical interventions are, however, rarely performed in the cervical and the interscapular regions in infancy (20), limiting the possibility of performing a comparative analysis. The developmental origin of BAT and BeAT is distinct $(18,19)$, and our study also suggests that AKGs lack an effect on BAT. This may be due to the scarcity of ATMs in BAT (41) or, alternatively, to differences in AKG metabolism in BAT, WAT, and BeAT. A negative correlation between BeAT content and obesity has been shown in children (20). Follow-up of the patients enrolled in our study can provide valuable information on whether this negative correlation is a late-acting effect of insufficient breastfeeding. In addition, a recent study suggests that breastfeeding protects infants from the development of metabolic diseases through the establishment of a healthy intestinal microbiota (93). Because AKGs increase Lactobacillus proliferation (94), they might also contribute to this indirect metabolic effect of breastfeeding.

Today nearly $60 \%$ of infants are never breastfed $(95,96)$ and childhood obesity may have its origins, at least in part, in insufficient breastfeeding $(6-8,97)$. Components of the manufactured infant formula might also be responsible for the negative metabolic impact of the absence of breastfeeding (98). Moreover, certain miRNA species enriched in cow milk - the main component of infant formula - inhibit BeAT development (99). Against this background, we show that maternal AKGs are the key signals for healthy BeAT development and that ATMs play an essential role in mediating this mother-to-child lipid signaling.

\section{Methods}

Animals. We used C57BL/6, male Lepr ${ }^{d b / d b}$ mice (Charles River Laboratories) and CD1 mice (CLEA Japan Inc.). Ptafr ${ }^{--}$and Pla2g2a-Tg mice were developed as described previously $(49,77)$. For treatments, we used AKGs (CA and SA from Nippon Chemicals; BA from Merck Sigma-Aldrich), dissolved in AKG-free sterile olive oil. Mice were treated daily with vehicle or AKGs by oral administration. We used littermates for vehicle and AKG treatment. To induce obesity, we used a HFD as described previously (62), and AKGs were provided in the diet for the treatment group. To deplete ATMs, we used intraperitoneal injection of clodronate-filled liposomes (Clodronate Liposomes) as described previously (100). As a control, we used PBS-filled liposomes. AT samples from nonhuman primate Macaca mulatta infants $(n=3)$ were provided by the Wisconsin National Primate Research Center (Madison, Wisconsin, USA). Canine breast milk samples were provided by a veterinary clinic in Budapest; goat and cow milk were purchased from commercial sources.

Human samples. Subcutaneous iAT from human infants was collected in the Leipzig Childhood Adipose Tissue cohort during elective surgery (42) (ClinicalTrials.gov NCT02208141). Infants were assigned to breastfed or formula-fed groups after ROC analysis (Supplemental Figure 6A). Duration of breastfeeding (as a percentage of age) was used as a predictor variable, and the optimal cut-off for group assignment was estimated by means of the Youden index using the Optimal Cutpoints package of R software (www.r-project.org). For all children included in the study, written informed consent was obtained from the parents.

Cell culture and FACS. Primary mouse and human ATMs, preadipocytes, and adipocytes were isolated by collagenase digestion and 
separation of cell fractions and subsequently analyzed or cultured as described before $(41,62,101)$. The 3T3-L1 preadipocyte cell line was obtained from ATCC and maintained and differentiated into adipocytes as described before (102). Cells were maintained in 6-well plates and 96-well plates for in-cell ELISA or, for fluorescent microscopy, on optical transparent glass-bottom plates (Greiner Bio-One GmbH). J774A.1 mouse macrophages and THP-1 human monocytic cell lines were obtained from ATCC, maintained, and checked regularly for Mycoplasma, as previously described (62). Phagocytosis of macrophages was tested by fluorescent latex beads, as described before (103). Preparative cell sorting and FACS analysis of ATMs was described before (62). Human ATMs were defined as CD68 ${ }^{+}$, mouse ATMs as $\mathrm{F} 4 / 80^{+}$and $\mathrm{CD} 11 \mathrm{~b}^{+}$. Mitochondrial content was analyzed with MitoTracker dyes (Thermo Fisher) according to the manufacturer's instruction. MTY was kindly provided by Y.-T. Chang (Pohang University of Science and Technology, Republic of Korea). Flow repository identifiers of FACS data were as follows: FR-FCM-ZYPU, FR-FCM-ZYXH, FR-FCM-ZY9E, and FR-FCM-ZYUU.

$m R N A$ analysis and NGS. Extraction of total RNA was described previously (62). qPCR assays were carried out using ViiA7 (Thermo Fisher) and Quant-Studio-3 (Applied Biosystems) equipment, using mouse Bactin and Ppia and human ACTB, TBP and HPRT1 as reference. Primer sequences are provided in Supplemental Table 3. NGS analysis was carried out on the BGISEQ-500 platform by BGI Genomic Services, generating on average about 26.20 M reads per sample. After reads filtering, clean reads were mapped to the reference genome using HISAT. On average, 96.09\% reads were mapped (Supplemental Figure 16, A and B), and clean reads were mapped to reference transcripts using Bowtie2, then gene expression levels for each sample were calculated with RSE. For annotation of transcripts we used EnrichR $(104,105)$ and Interferome 2.0 (106); clustered image maps (CIMs) were rendered by CIMminer (107). NGS data were deposited in the NCBI's Gene Expression Omnibus database (GEO GSE125405).

Histology and image analysis. Tissues were fixed in 4\% PFA and embedded into paraffin as described (62). Immunohistochemistry against UCP1 and F4/80 antigen was performed on paraffin-embedded sections (Supplemental Figure 16, C-F). Antibodies are listed in Supplemental Table 3. H\&E and PAS staining were performed according to the manufacturer's instructions (Carl Roth). BeAT area was measured with our custom-designed software; adipocyte size was measured with Image (NIH) (Supplemental Figure 16, G and H).

Supplemental methods. AKG determination, AR, ELISAs, overexpression studies, indirect calorimetry, the oxygen consumption test, Western blotting, and TEM analysis are detailed in Supplemental Methods and Supplemental Tables 4-6. Full, uncut gels are available in the supplemental material.
Statistics. Data are represented as mean \pm SEM, along with each individual data point. When data are represented as CIMs to visualize gene transcription differences among experimental conditions, we indicate transcript levels relative to reference genes, fold changes evoked by specific treatments, or Z-scores of the relative abundance of the transcripts, as indicated in the figures. The applied statistical tests are indicated in the figure legends; $P<0.05$ was considered significant.

Study approval. Human study protocols were approved by the ethics committees of the University of Leipzig (265-08-ff; NCT02208141) and the University of Ulm $(79 / 18,368 / 18)$. Adult AT was collected for a previous study (62) and under ethical approval of the University of Hannover, Hannover, Germany (3475-2017). Animal experiments were approved by local ethical committees (NPHC, PE/EA/20101/2016; Regional Council, Tübingen, Germany, 1206, O.189-20, O.232-1, 1398, O.232-2, 1441; Azabu University, 180112-3; University of Tennessee IACUC, 18-019.0-A; Wright State University IACUC, 1075).

\section{Author contributions}

HY, SD, JC, ACH, VD, A Harauma, YH, TM, KL, CL, LB, JT, MSK, and CR carried out experiments. AK, SB, EAP, JBT, and A Herwig designed experiments. GK designed and validated automated image analysis. TR conceived the project, designed experiments, and wrote the manuscript.

\section{Acknowledgments}

This study was supported by the German Research Fund (RO4856/1-2 to TR; CRC1052 C05 to AK), the European Foundation for the Study of Diabetes on New Targets for Type 2 Diabetes, supported by MSD (96403 to TR); the Federal Ministry of Education and Research, Germany (FKZ, 01EO1501 IFB Adiposity Diseases to AK); Horizon 2020 Research and Innovation Program MCIF (655598 to TR); and the PRC Scholarship Council (201808080208 to HY). Nonhuman primate tissues were provided by the Wisconsin National Primate Research Center Nonhuman Primate Biological Materials Distribution Core (award no. P51OD011106). Indirect calorimetry equipment was developed and provided by Gerhard Heldmaier. AT samples were kindly provided by Sarah Strauß (Hannover Medical University). The help of Benjamin Mayer with statistical analyses and the contributions of S.F.H. Waqas, Grace Ampem, and Kristina Geiß are appreciated. We thank Kenneth McCreath for scientific editing. The authors are grateful for the support of Harald Wolf, University of Ulm.

Address correspondence to: Tamás Röszer, Institute of Neurobiology, University of Ulm Albert-Einstein-Allee 11, 89081 Ulm, Germany. Phone: 49.0.731.50.22695; Email: tamas.roeszer@uni-ulm.de.
1. Rosen ED, Spiegelman BM. What we talk about when we talk about fat. Cell. 2014;156(1-2):20-44.

2. Nishtar S, Gluckman P, Armstrong T. Ending childhood obesity: a time for action. Lancet. 2016;387(10021):825-827.

3. Geserick M, et al. Acceleration of BMI in early childhood and risk of sustained obesity. $N$ Engl J Med. 2018;379(14):1303-1312.

4. Hidayat K, Du X, Shi BM. Body fatness at a young age and risks of eight types of cancer: systematic review and meta-analysis of observational stud- ies. Obes Rev. 2018;19(10):1385-1394.

5. [No authors listed]. Global report on diabetes. World Health Organization. https://www.who. int/diabetes/global-report/en/. Accessed April 12, 2019.

6. Uwaezuoke SN, Eneh CI, Ndu IK. Relationship between exclusive breastfeeding and lower risk of childhood obesity: a narrative review of published evidence. Clin Med Insights Pediatr. 2017;11:1179556517690196.

7. Bardanzellu F, Fanos V, Reali A. "Omics” in human colostrum and mature milk: looking to old data with new eyes. Nutrients. 2017;9(8):E843.

8. Yan J, Liu L, Zhu Y, Huang G, Wang PP. The association between breastfeeding and childhood obesity: a meta-analysis. BMC Public Health. 2014;14:1267.

9. Savino F, Fissore MF, Liguori SA, Oggero R. Can hormones contained in mothers' milk account for the beneficial effect of breast-feeding on obesity in children? Clin Endocrinol (Oxf). 
2009;71(6):757-765.

10. Koletzko B. Human milk lipids. Ann Nutr Metab. 2016;69(Suppl 2):28-40.

11. Boutens L, Stienstra R. Adipose tissue macrophages: going off track during obesity. Diabetologia. 2016;59(5):879-894.

12. Insull W, Hirsch J, James T, Ahrens EH. The fatty acids of human milk. II. Alterations produced by manipulation of caloric balance and exchange of dietary fats. J Clin Invest. 1959;38(2):443-450.

13. Cinti S. Adipose organ development and remodeling. Compr Physiol. 2018;8(4):1357-1431.

14. Hamosh M. Protective function of proteins and lipids in human milk. Biol Neonate. 1998;74(2):163-176.

15. Hartvigsen K, et al. 1-O-alkyl-2-(omega-oxo) acyl-sn-glycerols from shark oil and human milk fat are potential precursors of PAF mimics and GHB. Lipids. 2006;41(7):679-693.

16. Wan Y, Saghatelian A, Chong LW, Zhang CL, Cravatt BF, Evans RM. Maternal PPAR gamma protects nursing neonates by suppressing the production of inflammatory milk. Genes Dev. 2007;21(15):1895-1908.

17. van Elten TM, et al. Breast milk fatty acid composition has a long-term effect on the risk of asthma, eczema, and sensitization. Allergy. 2015;70(11):1468-1476.

18. Ikeda K, Maretich P, Kajimura S. The common and distinct features of brown and beige adipocytes. Trends Endocrinol Metab. 2018;29(3):191-200.

19. Symonds ME, Bloor I, Ojha S, Budge H. The placenta, maternal diet and adipose tissue development in the newborn. Ann Nutr Metab. 2017;70(3):232-235.

20. Rockstroh D, et al. Direct evidence of brown adipocytes in different fat depots in children. PLoS ONE. 2015;10(2):e0117841.

21. Sidossis L, Kajimura S. Brown and beige fat in humans: thermogenic adipocytes that control energy and glucose homeostasis. JClin Invest. 2015;125(2):478-486.

22. Qian L, et al. Determination of alkylglycerol contents in breast milk [Chinese]. J Clin Pediatr. 2014;32(6):540-543.

23. Hallgren B, Niklasson A, Ställberg G, Thorin H. On the occurrence of 1-O-alkylglycerols and 1-O-(2-methoxyalkyl)glycerols in human colostrum, human milk, cow's milk, sheep's milk, human red bone marrow, red cells, blood plasma and a uterine carcinoma. Acta Chem Scand, B, Org Chem Biochem. 1974;28(9):1029-1034.

24. Ahrné L, Björck L, Claesson O. Alkylglycerols in bovine colostrum and milk in relation to precursor levels. J Dairy Res. 1983;50(2):201-206.

25. Oh SY, Jadhav LS. Effects of dietary alkylglycerols in lactating rats on immune responses in pups. Pediatr Res. 1994;36(3):300-305.

26. Watschinger K, et al. Tetrahydrobiopterin and alkylglycerol monooxygenase substantially alter the murine macrophage lipidome. Proc Natl Acad Sci U S A. 2015;112(8):2431-2436.

27. Tokuoka SM, Kita Y, Shindou H, Shimizu T. Alkylglycerol monooxygenase as a potential modulator for PAF synthesis in macrophages. Biochem Biophys Res Commun. 2013;436(2):306-312.

28. Watschinger K, Werner ER. Orphan enzymes in ether lipid metabolism. Biochimie.
2013;95(1):59-65.

29. Frühbeck G. Overview of adipose tissue and its role in obesity and metabolic disorders. Methods Mol Biol. 2008;456:1-22.

30. Shin $\mathrm{H}$, et al. Lipolysis in brown adipocytes is not essential for cold-induced thermogenesis in mice. Cell Metab. 2017;26(5):764-777.e5.

31. Wu J, et al. Beige adipocytes are a distinct type of thermogenic fat cell in mouse and human. Cell. 2012;150(2):366-376.

32. Qiu Y, et al. Eosinophils and type 2 cytokine signaling in macrophages orchestrate development of functional beige fat. Cell. 2014;157(6):1292-1308 .

33. Fischer K, et al. Alternatively activated macrophages do not synthesize catecholamines or contribute to adipose tissue adaptive thermogenesis. Nat Med. 2017;23(5):623-630.

34. Pirzgalska RM, et al. Sympathetic neuron-associated macrophages contribute to obesity by importing and metabolizing norepinephrine. Nat Med. 2017;23(11):1309-1318.

35. Sharma LK, Lu J, Bai Y. Mitochondrial respiratory complex I: structure, function and implication in human diseases. Curr Med Chem. 2009;16(10):1266-1277.

36. Babaei R, et al. Jak-TGF $\beta$ cross-talk links transient adipose tissue inflammation to beige adipogenesis. Sci Signal. 2018;11(527):eaai7838.

37. Arai S, et al. Mitochondria-targeted fluorescent thermometer monitors intracellular temperature gradient. Chem Commun (Camb). 2015;51(38):8044-8047.

38. Lane N. Hot mitochondria? PLoS Biol. 2018;16(1):e2005113.

39. Chrétien D, et al. Mitochondria are physiologically maintained at close to $50^{\circ} \mathrm{C}$. PLoS Biol. 2018;16(1):e2003992.

40. Russo L, Lumeng CN. Properties and functions of adipose tissue macrophages in obesity. Immunology. 2018;155(4):407-417.

41. Hassnain Waqas SF, et al. Adipose tissue macrophages develop from bone marrow-independent progenitors in. JLeukoc Biol. 2017;102(3):845-855.

42. Landgraf $K$, et al. Evidence of early alterations in adipose tissue biology and function and its association with obesity-related inflammation and insulin resistance in children. Diabetes. 2015;64(4):1249-1261.

43. Röszer T. Understanding the mysterious M2 macrophage through activation markers and effector mechanisms. Mediators Inflamm. 2015;2015:816460.

44. Ginhoux F, Schultze JL, Murray PJ, Ochando J, Biswas SK. New insights into the multidimensional concept of macrophage ontogeny, activation and function. Nat Immunol. 2016;17(1):34-40.

45. Hichami A, et al. Modulation of platelet-activating-factor production by incorporation of naturally occurring 1-O-alkylglycerols in phospholipids of human leukemic monocyte-like THP-1 cells. Eur J Biochem. 1997;250(2):242-248.

46. Detopoulou P, et al. The relation of diet with PAF and its metabolic enzymes in healthy volunteers. Eur J Nutr. 2015;54(1):25-34

47. Davies SS, et al. Oxidized alkyl phospholipids are specific, high affinity peroxisome proliferator-activated receptor gamma ligands and agonists. J Biol Chem. 2001;276(19):16015-16023.
48. Gountopoulou A, Leondaritis G, Galanopoulou $\mathrm{D}$, Mavri-Vavayanni M. TNFalpha is a potent inducer of platelet-activating factor synthesis in adipocytes but not in preadipocytes. Differential regulation by PI3K. Cytokine. 2008;41(2):174-181.

49. Kuefner MS, et al. Secretory phospholipase A. JLipid Res. 2017;58(9):1822-1833.

50. Curfs DM, et al. Macrophage secretory phospholipase $\mathrm{A} 2$ group $\mathrm{X}$ enhances anti-inflammatory responses, promotes lipid accumulation, and contributes to aberrant lung pathology. J Biol Chem. 2008;283(31):21640-21648.

51. Hasegawa S, et al. Role of PAF receptor in proinflammatory cytokine expression in the dorsal root ganglion and tactile allodynia in a rodent model of neuropathic pain. PLoS One. 2010;5(5):e10467.

52. Murase R, et al. Group X secreted phospholipase A2 releases $\omega 3$ polyunsaturated fatty acids, suppresses colitis, and promotes sperm fertility. J Biol Chem. 2016;291(13):6895-6911.

53. García-Alonso V, Clària J. Prostaglandin E2 signals white-to-brown adipogenic differentiation. Adipocyte. 2014;3(4):290-296.

54. Yamaguchi M, et al. A platelet-activating factor (PAF) receptor deficiency exacerbates dietinduced obesity but PAF/PAF receptor signaling does not contribute to the development of obesity-induced chronic inflammation. Biochem Pharmacol. 2015;93(4):482-495.

55. Menezes-Garcia Z, et al. Lack of platelet-activating factor receptor protects mice against dietinduced adipose inflammation and insulinresistance despite fat pad expansion. Obesity (Silver Spring). 2014;22(3):663-672.

56. Marques SA, et al. The platelet-activating factor receptor activates the extracellular signal-regulated kinase mitogen-activated protein kinase and induces proliferation of epidermal cells through an epidermal growth factor-receptor-dependent pathway. J Pharmacol Exp Ther. 2002;300(3):1026-1035.

57. Cao W, et al. p38 mitogen-activated protein kinase is the central regulator of cyclic AMP-dependent transcription of the brown fat uncoupling protein 1 gene. Mol Cell Biol. 2004;24(7):3057-3067.

58. Maurer SF, Fromme T, Grossman LI, Hüttemann $\mathrm{M}$, Klingenspor $\mathrm{M}$. The brown and brite adipocyte marker Cox7a1 is not required for non-shivering thermogenesis in mice. Sci Rep. 2015;5:17704.

59. Rong JX, et al. Adipose mitochondrial biogenesis is suppressed in $\mathrm{db} / \mathrm{db}$ and high-fat diet-fed mice and improved by rosiglitazone. Diabetes. 2007;56(7):1751-1760.

60. Hui X, et al. Adiponectin enhances cold-induced browning of subcutaneous adipose tissue via promoting M2 macrophage proliferation. Cell Metab. 2015;22(2):279-290.

61. Amano SU, et al. Local proliferation of macrophages contributes to obesity-associated adipose tissue inflammation. Cell Metab. 2014;19(1):162-171.

62. Waqas SFH, et al. Neuropeptide FF increases M2 activation and self-renewal of adipose tissue macrophages. J Clin Invest. 2017;127(7):2842-2854.

63. Dhir A, et al. Mitochondrial double-stranded 
RNA triggers antiviral signalling in humans. Nature. 2018;560(7717):238-242.

64. Zhong Z, et al. New mitochondrial DNA synthesis enables NLRP3 inflammasome activation. Nature. 2018;560(7717):198-203.

65. Lu X, et al. Mitophagy controls beige adipocyte maintenance through a Parkin-dependent and UCP1-independent mechanism. Sci Signal. 2018;11(527):eaap8526.

66. Lu Y, et al. Mitophagy is required for brown adipose tissue mitochondrial homeostasis during cold challenge. Sci Rep. 2018;8(1):8251.

67. Coats BR, et al. Metabolically activated adipose tissue macrophages perform detrimental and beneficial functions during diet-induced obesity. Cell Rep. 2017;20(13):3149-3161.

68. Ahrné L, Palmquist DL. Incorporation of [hydrogen-3] alkylglycerolether and [carbon-14] hexadecanol into bovine colostrum and milk lipids. J Dairy Sci. 1982;65(10):1905-1911.

69. Lodhi IJ, et al. Inhibiting adipose tissue lipogenesis reprograms thermogenesis and PPAR $\gamma$ activation to decrease diet-induced obesity. Cell Metab. 2012;16(2):189-201.

70. Dahlman I, et al. A common haplotype in the G-protein-coupled receptor gene GPR74 is associated with leanness and increased lipolysis. Am J Hum Genet. 2007;80(6):1115-1124.

71. van Harmelen V, et al. Effects of pain controlling neuropeptides on human fat cell lipolysis. Int $J$ Obes (Lond). 2010;34(8):1333-1340.

72. Rosa-Caldwell ME, et al. Autophagy activation, not peroxisome proliferator-activated receptor $\gamma$ coactivator $1 \alpha$, may mediate exercise-induced improvements in glucose handling during diet-induced obesity. Exp Physiol. 2017;102(9):1194-1207.

73. Singh R, et al. Autophagy regulates adipose mass and differentiation in mice. JClin Invest. 2009;119(11):3329-3339.

74. Odegaard JI, Ganeshan K, Chawla A. Adipose tissue macrophages: Amicus adipem? Cell Metab. 2013;18(6):767-768.

75. Santiago HC, et al. Platelet activating factor receptor-deficient mice present delayed interferongamma upregulation and high susceptibility to Leishmania amazonensis infection. Microbes Infect. 2006;8(11):2569-2577.

76. Qian L, Zhang M, Wu S, Zhong Y, Van Tol E, Cai W. Alkylglycerols modulate the proliferation and differentiation of non-specific agonist and specific antigen-stimulated splenic lymphocytes. PLoS One. 2014;9(4):e96207.

77. Sugatani J, et al. Antiobese function of plateletactivating factor: increased adiposity in plateletactivating factor receptor-deficient mice with age. FASEB J. 2014;28(1):440-452.
78. Filgueiras LR, et al. PAFR in adipose tissue macrophages is associated with anti-inflammatory phenotype and metabolic homoeostasis. Clin Sci. 2016;130(8):601-612.

79. Okada T, Miyashita M, Kuromori Y, Iwata F, Harada K, Hattori H. Platelet-activating factor acetylhydrolase concentration in children with abdominal obesity. Arterioscler Thromb Vasc Biol. 2006;26(5):e40-e41.

80. Chroni A, Mavri-Vavayanni M. Characterization of a platelet activating factor acetylhydrolase from rat adipocyte. Life Sci. 2000;67(23):2807-2825.

81. Matthews VB, et al. Interleukin-6-deficient mice develop hepatic inflammation and systemic insulin resistance. Diabetologia. 2010;53(11):2431-2441.

82. Wan Z, Perry CG, Macdonald T, Chan CB, Holloway GP, Wright DC. IL-6 is not necessary for the regulation of adipose tissue mitochondrial content. PLoS One. 2012;7(12):e51233.

83. Ellingsgaard $\mathrm{H}$, et al. Interleukin- 6 enhances insulin secretion by increasing glucagon-like peptide-1 secretion from L cells and alpha cells. Nat Med. 2011;17(11):1481-1489.

84. Timper K, et al. IL-6 improves energy and glucose homeostasis in obesity via enhanced central IL-6 trans-signaling. Cell Rep. 2017;19(2):267-280.

85. Wernstedt Asterholm I, et al. Adipocyte inflammation is essential for healthy adipose tissue expansion and remodeling. Cell Metab. 2014;20(1):103-118.

86. Sun K, Gao Z, Kolonin MG. Transient inflammatory signaling promotes beige adipogenesis. $\mathrm{Sci}$ Signal. 2018;11(527):eaat3192.

87. Nguyen KD, et al. Alternatively activated macrophages produce catecholamines to sustain adaptive thermogenesis. Nature. 2011;480(7375):104-108.

88. Finlin BS, et al. Mast cells promote seasonal white adipose beiging in humans. Diabetes. 2017;66(5):1237-1246

89. Ortega Martinez de Victoria E, et al. Macrophage content in subcutaneous adipose tissue: associations with adiposity, age, inflammatory markers, and whole-body insulin action in healthy Pima Indians. Diabetes. 2009;58(2):385-393.

90. Alexander G, Bennett JW, Gemmell RT. Brown adipose tissue in the new-born calf (Bos taurus). JPhysiol (Lond). 1975;244(1):223-234.

91. Lidell ME, et al. Evidence for two types of brown adipose tissue in humans. Nat Med. 2013;19(5):631-634.

92. Gilsanz V, Hu HH, Kajimura S. Relevance of brown adipose tissue in infancy and adolescence. Pediatr Res. 2013;73(1):3-9.

93. Stewart CJ, et al. Temporal development of the gut microbiome in early childhood from the TEDDY study. Nature. 2018;562(7728):583-588.
94. Chorostowska-Wynimko J, Krotkiewski M, Radomska-Leśniewska D, Sokolnicka I, Skopińska-Rózewska E. The synergistic effect of lactic acid bacteria and alkylglycerols on humoral immunity in mice. Int J Tissue React. 2001;23(3):81-87.

95. Grummer-Strawn LM, et al. New World Health Organization guidance helps protect breastfeeding as a human right. Matern Child Nutr. 2017;13(4):e12491.

96. Biggs KV, Hurrell K, Matthews E, Khaleva E, Munblit D, Boyle RJ. Formula milk supplementation on the postnatal ward: a cross-sectional analytical study. Nutrients. 2018;10(5):E608.

97. Mannan H. Early infant feeding of formula or solid foods and risk of childhood overweight or obesity in a socioeconomically disadvantaged region of Australia: a longitudinal cohort analysis. Int J Environ Res Public Health. 2018;15(8):E1685.

98. Scopesi F, et al. 7-ketocholesterol in human and adapted milk formulas. Clin Nutr. 2002;21(5):379-384.

99. Melnik BC, John SM, Schmitz G. Milk is not just food but most likely a genetic transfection system activating mTORC1 signaling for postnatal growth. Nutr J. 2013;12:103

100.Bu L, Gao M, Qu S, Liu D. Intraperitoneal injection of clodronate liposomes eliminates visceral adipose macrophages and blocks high-fat dietinduced weight gain and development of insulin resistance. AAPS J. 2013;15(4):1001-1011.

101.Hausman DB, Park HJ, Hausman GJ. Isolation and culture of preadipocytes from rodent white adipose tissue. Methods Mol Biol. 2008;456:201-219.

102.Zebisch K, Voigt V, Wabitsch M, Brandsch M. Protocol for effective differentiation of 3T3-L1 cells to adipocytes. Anal Biochem. 2012;425(1):88-90.

103. Röszer T, et al. Autoimmune kidney disease and impaired engulfment of apoptotic cells in mice with macrophage peroxisome proliferatoractivated receptor gamma or retinoid $\mathrm{X}$ receptor alpha deficiency. J Immunol. 2011;186(1):621-631.

104.Chen EY, et al. Enrichr: interactive and collaborative HTML5 gene list enrichment analysis tool. BMC Bioinformatics. 2013;14:128.

105.Kuleshov MV, et al. Enrichr: a comprehensive gene set enrichment analysis web server 2016 update. Nucleic Acids Res. 2016;44(W1):W90-W97.

106. Rusinova I, et al. Interferome v2.0: an updated database of annotated interferon-regulated genes. Nucleic Acids Res. 2013; 41(Database issue):D1040-D1046.

107. Weinstein JN, et al. An information-intensive approach to the molecular pharmacology of cancer. Science. 1997;275(5298):343-349. 